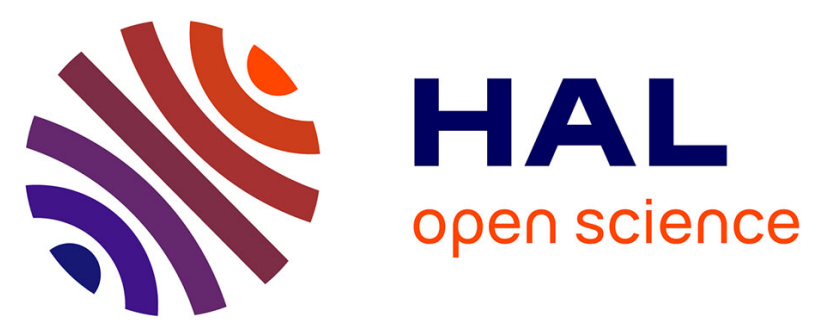

\title{
Chapitre III - Les pratiques postcrématoires dans les bûchers
}

\author{
Frédérique Blaizot, Valérie Bel, Christine Bonnet, Patrice \\ Georges-Zimmermann, Anne Richier
}

\section{To cite this version:}

Frédérique Blaizot, Valérie Bel, Christine Bonnet, Patrice Georges-Zimmermann, Anne Richier. Chapitre III - Les pratiques postcrématoires dans les bûchers. Gallia - Archéologie de la France antique, 2009, Pratiques et espaces funéraires de la Gaule durant l'Antiquité, 66 (1), pp.151-174. 10.3406/galia.2009.3374 . hal-01911009

\section{HAL Id: hal-01911009 \\ https://hal.science/hal-01911009}

Submitted on 7 Jan 2020

HAL is a multi-disciplinary open access archive for the deposit and dissemination of scientific research documents, whether they are published or not. The documents may come from teaching and research institutions in France or abroad, or from public or private research centers.
L'archive ouverte pluridisciplinaire HAL, est destinée au dépôt et à la diffusion de documents scientifiques de niveau recherche, publiés ou non, émanant des établissements d'enseignement et de recherche français ou étrangers, des laboratoires publics ou privés.

\section{(ㅇ)(1) $\$$}

Distributed under a Creative Commons Attribution - NonCommercial - NoDerivatives| 4.0 


\title{
LES PRATIQUES POSTCRÉMATOIRES DANS LES BÛCHERS
}

\author{
Frédérique BLAIzOT, Valérie BEL, Christine BOnNET, Patrice GEORGES et Anne RICHIER
}

\section{LES PRÉLÈVEMENTS D'OSSEMENTS}

\section{LES DONNÉES OSTÉOLOGIQUES}

Une fois le corps détruit par le feu, différents gestes sont accomplis dans les bûchers en fosse. Parmi les interventions postcrématoires, le prélèvement d'une partie plus ou moins importante de la masse osseuse, destiné à reconstituer la structure du dépôt définitif des restes, peut être mis en évidence par les études ostéologiques : celles-ci révèlent des déficits dans la quantité d'ossements en présence et permettent de définir les modalités du prélèvement. Ces études quantitatives sont fondées sur la masse osseuse qui est comparée à des données théoriques recueillies en contexte expérimental, notamment celles fournies par Herrmann, 1976 et MacKinley, 1993 (tabl. XVIIIa). Celles-ci indiquent que le squelette complet d'un adulte calciné pèse au minimum $1000 \mathrm{~g}$. En raison de la fragmentation et de la survie différentielles des régions anatomiques, un simple dénombrement des fragments ne permet pas d'évaluer la représentation du squelette, puisque la part réelle de chaque os parmi l'ensemble des fragments recueillis est minimisée du fait des esquilles inférieures ou égales à $2 \mathrm{~mm}$ qui ne peuvent être identifiées précisément (Duday, 1987). Une masse osseuse archéologique qui s'inscrit dans la marge de variation des valeurs théoriques, indique que la totalité ou la quasi-totalité du squelette se trouve dans la structure, à condition bien sûr que la couche de crémation soit intégralement conservée. En revanche, une masse nettement inférieure à la valeur la plus faible signifie que le squelette a été en partie ramassé, tandis qu'une masse bien supérieure à la valeur la plus forte laisse envisager que l'on a plus d'un individu.

Deux précisions doivent toutefois être apportées. La première est que l'on ne dispose de très peu de références pour les individus immatures (Trotter, Hixon, 1974 ; Warren et al., 1997, p. 420) (tabl. XVIIIb). La deuxième est que la méthode ne permet que de distinguer les cas les plus évidents : en effet, la variabilité du poids d'un squelette est telle qu'un prélèvement de quelques dizaines de grammes ne va pas apparaître, tandis qu'une masse osseuse inférieure à la valeur théorique la plus haute peut très bien correspondre à deux individus dont le stade de maturation est identique et pour lesquels aucun doublet n'a été reconnu. Dans ce dernier cas, l'examen des indices pondéraux par région anatomique (pourcentage de telle ou telle région anatomique sur la masse totale du squelette, $c f$. Duday, 1987 ; Duday et al., 2000) peut apporter des arguments en faveur de telle ou telle interprétation ; par exemple, une surreprésentation extrême, illustrée par des valeurs brutes très élevées, conduit tout naturellement à s'interroger sur le nombre de sujets.

L'examen de la part relative prise par les différentes régions anatomiques dans la masse d'ossements (indices pondéraux) contenus dans une structure permet d'approcher les modalités du prélèvement avec un peu plus de précision. Les tables de références publiées par Krogman 
Tabl. XVIII - Données théoriques des masses osseuses : a, sujets adultes selon Herrmann (1976) et MacKinley (1993); b, sujets immatures selon Trotter et Hixon (1974).

\begin{tabular}{|c|c|c|c|}
\hline Sujets adultes & Masse min. (en g) & Masse max. (en g) & Moyenne \\
\hline Herrmann, 1976 & 970 & 2630 & 1770 \\
\hline MacKinley, 1993 & 1001,5 & 2422,5 & 1625 \\
\hline
\end{tabular}

a

\begin{tabular}{|c|c|c|}
\hline $\begin{array}{c}\text { Sujets immatures } \\
\text { Trotter, Hixon, 1974 }\end{array}$ & $\begin{array}{c}\text { Masse minimale } \\
\text { moyenne (en g) }\end{array}$ & $\begin{array}{c}\text { Masse maximale } \\
\text { moyenne (en g) }\end{array}$ \\
\hline$<0,5$ ans & 71 & 95 \\
\hline $0,5-3$ ans & 268,4 & 315,8 \\
\hline $3-13$ ans & 932,6 & 1132,4 \\
\hline $13-25$ ans & 2724,3 & 4004,4 \\
\hline
\end{tabular}

b

(1978) indiquent la masse moyenne pour chaque pièce osseuse, assortie d'un écart-type permettant de calculer les marges de variation (tabl. XIX). Cependant, ces tables n'ont été établies que sur des squelettes adultes et sur des os non brûlés ; or, non seulement la survie des os brûlés dans la terre paraît varier selon les régions anatomiques (Blaizot, 2005), mais la part de régions anatomiques évolue au cours de la croissance. On observe notamment que la surreprésentation du crâne caractérise les restes des sujets immatures (Duday, 1989 ; Taffanel et al., 1998 ; Blaizot, 2005). Ces paramètres (fragilité intrinsèque de telle ou telle région anatomique et âge au décès du sujet) doivent ainsi être pris en compte dans l'interprétation des indices pondéraux.

La masse osseuse relevée dans les bûchers en fosse est fréquemment déficitaire, ce qui est normal puisque a priori, cette structure est destinée à brûler le corps et non à le conserver. C'est ce qu'indiquent clairement les exemples de bûchers en fosse presque complètement vidés, comme ceux des sites des 77-79 rues Pierre-Audry (bûcher 1080) et de la Favorite à Lyon (Tranoy, 1995b, p. 679), où il ne restait sur le fond, plus ou moins rubéfié, que quelques rares artefacts calcinés, épars. À Saint-Paul-Trois-Châteaux, le bûcher 71 ne conservait plus que des lambeaux de résidus charbonneux avec de rares vestiges de mobilier brûlés qui ont pu être rapprochés des éléments placés dans une tombe avec ossuaire retrouvée dans le même enclos (Bel et al., 2002, p. 229). L'ensemble funéraire du 78 avenue Jean-Jaurès à Nîmes comporte trois fosses partiellement rubéfiées pratiquement dépourvues de résidus (dont le bûcher en coffrage de dalles 1004 déjà mentionné). Des exemples comparables sont attestés à Aspiran (bûcher 11104 ou 11190, cf. Thernot et al., 2004, p. 297 et p. 328-329), et à Aix-en-Provence (fosses de combustion 44 et 155 de la ZAC Sextius-Mirabeau, $c f$. Nin et al., 2006, p. 210-211, fig. 53-54). Les données récemment publiées du site rhénan de Krefeld-Gellep présentent les mêmes caractéristiques : la masse osseuse recueillie dans les 215 bûchers oscille entre $6 \mathrm{~g}$ et plus de $1200 \mathrm{~g}$, avec 17 cas où elle est inférieure à $20 \mathrm{~g}$ et 5 cas où elle dépasse les $800 \mathrm{~g}$ (Pirling, 2002, p. 502 et p. 504). Ce site, comme celui de Champ Bussière à Pont-du-Château, dans le Puy-de-Dôme, sont particulièrement éloquents ; dans le premier, le bûcher 5948 ne renferme plus que 238 g d'ossements dispersés dans la couche de crémation, mais à son extrémité nord, se trouve un vase ossuaire contenant $1212 \mathrm{~g}$ d'ossements (Pirling, 2002). Dans le second, deux bûchers, qui conservent respectivement une masse osseuse de 277,6 g et 428,5 g, sont accompagnés chacun d'un vase ossuaire installé dans une fosse creusée à côté (fig. 106).

En réalité,l'absence de prélèvement ne peutêtre envisagée que dans les cas où le bûcher renferme une masse osseuse nettement supérieure à la valeur théorique minimale de $1 \mathrm{~kg}$ pour des pesées portant sur un tri effectué à la maille de $2 \mathrm{~mm}$, et où un seul sujet est déterminé. Ce cas n'a toutefois été relevé nulle part dans les régions étudiées tandis que les bûchers qui comportent une masse d'au moins $2000 \mathrm{~g}$ sont exceptionnels : on en dénombre un exemplaire sur le site de Saint-Fréjus à Marennes (bûcher 6318 : 2 024,4 g), un sur le site de Sainte-Barbe à Marseille (bûcher 136 : $2043 \mathrm{~g}$ ), un à Nîmes (Magaille 1021 : 1989,4 g) et un à l'Estel à Vers-Pontdu-Gard (bûcher 10 : $1953 \mathrm{~g}$ ).

À Lyon, sur 49 bûchers complets dont les masses osseuses ont été calculées ${ }^{9}$, six seulement ont une masse totale compatible avec la valeur minimale attendue (tabl. XX) : 41 offrent une masse totale significativement inférieure à celle-ci et dans trois cas, on ne peut le déterminer (présence d'enfants ou masse tout juste acceptable pour un NMI de deux sujets, dont l'un est immature). Les bûchers font donc généralement l'objet de prélèvements importants, le cas étant particulièrement flagrant à la Favorite aux $\mathrm{I}^{\mathrm{er}}$ et $\mathrm{II}^{\mathrm{e}}$ s. (Blaizot, Tranoy, 2004) ${ }^{10}$. La masse osseuse relevée dans les 31 bûchers dont la fosse est conservée à plus de $85 \%$ varie en effet de $28,5 \mathrm{~g}$ à $853 \mathrm{~g}$, avec une moyenne de 326,8 g et un écart-type de 229,7. Le site de la rue des Granges, dont les sept bûchers datent du règne de Claude, montre une situation un peu différente :

9. Dans un très grand nombre de cas, la couche de crémation est incomplète du fait de l'érosion des sites, de destructions partielles a posteriori ou de l'inaccessibilité à l'ensemble de la structure.

10. Les chiffres présentés ici reposent sur un plus grand nombre de structures que ceux utilisés dans Blaizot, Tranoy, 2004 (prise en compte notamment des bûchers dont la couche est conservée entre $85 \%$ et $100 \%$ ). Les données brutes ont été produites par G. Grévin et P. Bailet, UMR 6130 du CNRS à Draguignan. 
Tabl. XIX - Projection des indices pondéraux d'un squelette d'une structure archéologique sur les valeurs de références publiées par Krogman (1978) : mise en évidence des distorsions.

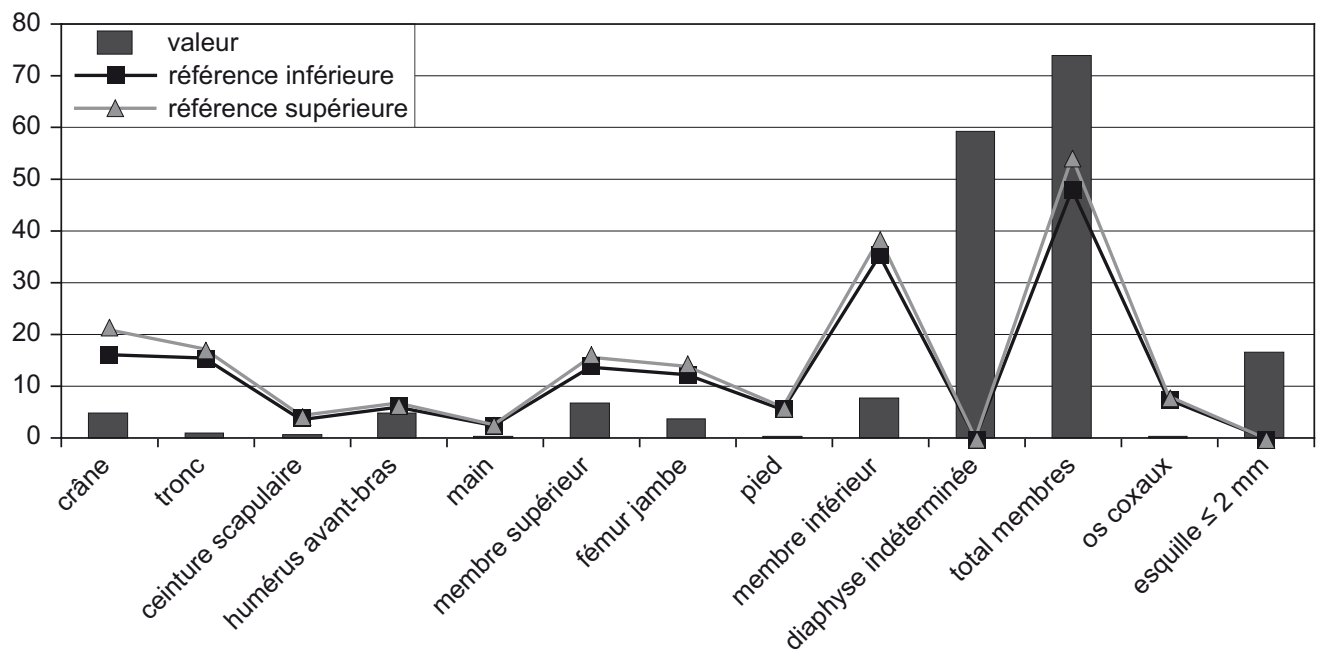

\begin{tabular}{|l|c|c|c|c|c|c|c|c|c|c|c|c|c|}
\hline \multicolumn{1}{|c|}{ Bûcher 147 } & Crâne & Tronc & $\begin{array}{c}\text { Ceinture } \\
\text { scapulaire }\end{array}$ & $\begin{array}{c}\text { Humérus } \\
\text { avant-bras }\end{array}$ & Main & $\begin{array}{c}\text { Membre } \\
\text { supérieur }\end{array}$ & $\begin{array}{c}\text { Fémur } \\
\text { jambe }\end{array}$ & Pied & $\begin{array}{c}\text { Membre } \\
\text { inférieur }\end{array}$ & $\begin{array}{c}\text { Diaphyse } \\
\text { indéterminée }\end{array}$ & $\begin{array}{c}\text { Total } \\
\text { membres }\end{array}$ & $\begin{array}{c}\text { Os } \\
\text { coxaux }\end{array}$ & $\begin{array}{c}\text { Esquille } \\
\mathbf{5} \mathbf{2} \text { mm }\end{array}$ \\
\hline Valeur & 4,8 & 1 & 0,7 & 4,9 & 0,3 & 6,8 & 3,7 & 0,3 & 7,7 & 59,3 & 74 & 0,3 & 16,6 \\
\hline Référence inférieure & 16,19 & 15,5 & 3,5 & 6 & 2,4 & 13,7 & 12,2 & 5,5 & 35,3 & 0 & 48 & 7,5 & 0 \\
\hline Référence supérieure & 21 & 17,3 & 4,3 & 6,8 & 2,6 & 15,7 & 13,9 & 6 & 38,7 & 0 & 54,4 & 8,1 & 0 \\
\hline
\end{tabular}

les trois fosses complètes renferment une masse osseuse acceptable (993 g, $1286 \mathrm{~g}$ et $1594 \mathrm{~g}$ ), de même qu'une quatrième pourtant incomplète $(1265 \mathrm{~g})$. Ces différences sont-elles d'ordre chronologique puisque les bûchers pris en compte pour la Favorite couvrent deux siècles, ce qui signifierait que la quantité d'ossements prélevés pour constituer la tombe évolue dans le temps ? À la Favorite, on remarque qu'aucun bûcher de la première moitié du $\mathrm{I}^{\mathrm{er}}$ s. apr. J.-C. ne conserve moins de 302 g et que quatre bûchers sur les cinq étudiés renferment une masse supérieure à 487 g. Cinq bûchers sur les dix-sept de la seconde moitié du $\mathrm{I}^{\mathrm{er}} \mathrm{s}$. et trois sur les neuf bûchers du $\mathrm{II}^{\mathrm{e}}$ s. renferment une masse supérieure à $302 \mathrm{~g}$. Le corpus est toutefois trop inégal pour en tirer des conclusions. À l'échelle de la série, on n'observe pas, en tout cas, de corrélation entre la longueur des bûchers (qui paraît diminuer dans le temps) et la masse osseuse conservée $(\mathrm{r}=0,142$ pour $25 \mathrm{ddl})$.

Les séries provençales (Vernègues, Aix-en-Provence, Marseille, auxquelles on a joint l'exemplaire de Bollène), comportent 62 bûchers complets pour lesquels l'étude pondérale a été réalisée. La masse moyenne des ossements brûlés conservés n'est que de 450,6 g et la variabilité est très importante (écart-type : 479,6). Six structures $\left(1 / 10^{\mathrm{e}}\right.$ ) seulement ont livré une masse supérieure à $1000 \mathrm{~g}$ et 27 structures ont fourni moins de $200 \mathrm{~g}$ d'os.
Dans le corpus méridional, la majorité des données provient du site des Communaux de Saint-Cézaire à Vernègues (Chapon et al., 2004), où les 48 bûchers ont livré des résultats particulièrement intéressants (tabl. XXI) : seuls trois d'entre eux semblent contenir les restes calcinés de sujets complets, et parmi eux, le bûcher 5 contient une masse exceptionnelle de $1774 \mathrm{~g}$ d'ossements pour un sujet unique. Le reste des bûchers contient des sujets incomplets, avec des masses totales s'échelonnant entre $0,1 \mathrm{~g}$ et $817 \mathrm{~g}$ et une moyenne générale de seulement $243 \mathrm{~g}$ (écart-type : 225). Des comparaisons entre les masses osseuses contenues dans les bûchers de Vernègues et leur répartition topochronologique mettent en évidence une grande variabilité pour toutes les fosses comprises entre le milieu du ${ }^{\mathrm{er}} \mathrm{s}$. (date du début de l'utilisation de l'ensemble funéraire) et le milieu du $\mathrm{II}^{\mathrm{e}} \mathrm{s}$. Les bûchers les plus tardifs, à partir de la seconde moitié $d u$ II $^{\mathrm{e}}$ s., illustrent des prélèvements en revanche moins importants, avec une moyenne osseuse de $698 \mathrm{~g}$ et un écart-type de 271. Les bûchers contenant moins de $200 \mathrm{~g}$ (soit plus de la moitié des structures) se répartissent dans toutes les zones et côtoient des bûchers plus riches en ossements, ce qui exclut l'hypothèse d'un déficit osseux dû à une destruction d'ordre taphonomique. Ainsi, à l'issue de la crémation, la quasi-totalité des bûchers de Vernègues a fait l'objet de prélèvements osseux, souvent très importants 


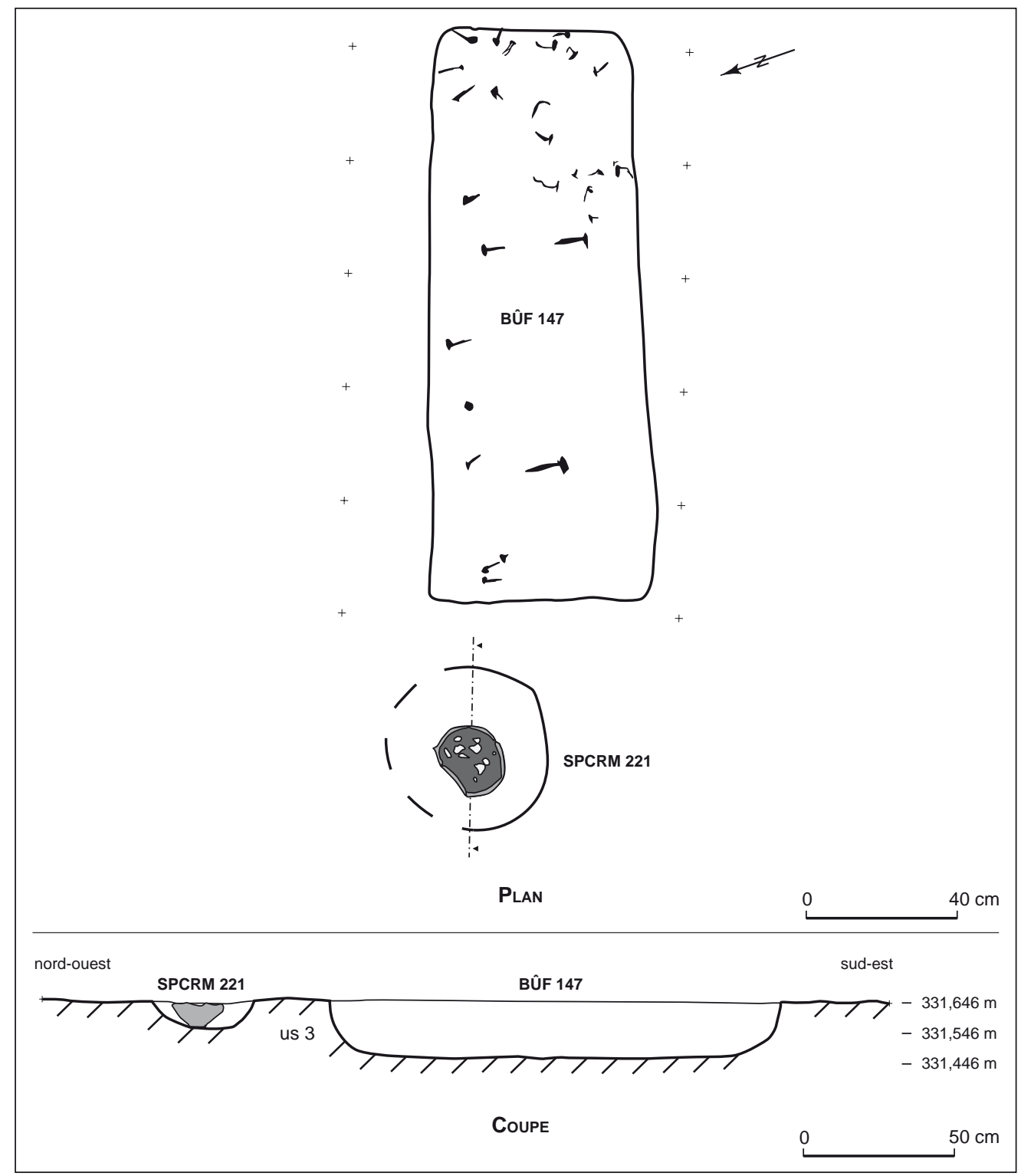

Fig. 106 - Dépôt de crémation en ossuaire associé au bûcher en fosse 147 de Champ Bussière à Pont-du-Château, Puy-de-Dôme (relevé : F. Blaizot, Inrap; DAO : D. Pelletier, Inrap).

et intéressant toutes les parties anatomiques des sujets, sans sélection. Ceci conduit tout naturellement à interpréter ces bûchers comme de simples fosses à crémation, les ossements prélevés ayant sans doute été transférés ailleurs pour constituer le lieu sépulcral. Un cas unique pourrait d'ailleurs l'attester : il s'agit d'un vase ossuaire contenu dans une petite fosse ovoïde (fosse 46) située dans une zone marginale du site, au-delà de laquelle plus aucune structure funéraire n'est présente. Ce vase, en céramique commune régionale, est couvert d'une tegula et contient 1212 g d'ossements (c'est-à-dire beaucoup plus d'ossements que dans l'écrasante majorité des bûchers), ainsi que du mobilier déposé dans la fosse. Cependant, aucune liaison secondaire ${ }^{11}$ n'a pu être établie entre les ossements de ce vase et ceux des bûchers. L'interprétation de ces bûchers à partir des résultats anthropologiques entre en contradiction avec certaines données archéologiques, qui pourraient conduire à identifier ces structures comme des sépultures plutôt que de simples bûchers. En effet, dans 7 cas, les fosses

11. Liaison secondaire : établie a posteriori par collage, appartenance à un même stade de maturation ou un même ensemble pathologique, etc. 
Tabl. XX - Masses osseuses relevées dans une sélection de bûchers lyonnais complets (d'après les données de J.-L. Gisclon, G. Grévin, J. Rouquet, É. Boës et C. Dumont).

\begin{tabular}{|c|c|c|c|}
\hline Localisation & $\mathbf{N}^{\circ}$ bûcher & Datation & $\begin{array}{l}\text { Masse osseuse brûlé } \\
\text { (en g, lots complets) }\end{array}$ \\
\hline Rue des Granges & 18 & milieu $\mathrm{I}^{\mathrm{er}} \mathrm{s}$. & 993,2 \\
\hline Rue des Granges & 22 & 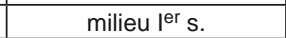 & 1286 \\
\hline Rue des Granges & 46 & milieu ler $\mathrm{s}$. & 1594 \\
\hline Rue du Commandant-Charcot & Les Roses, 1 & Augustéen & 436,1 \\
\hline Rue du Commandant-Charcot & Les Roses, 25 & Augustéen & 429,3 \\
\hline Avenue du Point-du-Jour & 1000 & fin $I^{\mathrm{er}}$-début $\mathrm{II}^{\mathrm{e}} \mathrm{s}$. & 719 \\
\hline 77-79, rue Pierre-Audry & 3041 & $2^{\mathrm{e}}$ quart $\mathrm{l}^{\mathrm{er}} \mathrm{s}$. & 642 \\
\hline 77-79, rue Pierre-Audry & 150 & $\left\|I^{e}-I\right\|^{e} s$ & 148 \\
\hline 77-79, rue Pierre-Audry & 159 & $\left\|I^{e}-I\right\|^{e} s$. & 13 \\
\hline 77-79, rue Pierre-Audry & 1093 & $\| I^{e}-I I^{e} s$. & 128 \\
\hline 54, rue Pierre-Audry & 22 & $2^{\mathrm{e}}$ moitié $\mathrm{I}^{\mathrm{er}} \mathrm{s}$.-début II $\mathrm{e}$. & 443 \\
\hline 54, rue Pierre-Audry & 30 & fin $l^{\mathrm{er}} \mathrm{s}$-début $\|^{\mathrm{e}} \mathrm{s}$. & 1069,2 \\
\hline 54, rue Pierre-Audry & 56 & fin ${ }^{\mathrm{er}} \mathrm{s}$ - -début $\|^{\mathrm{e}} \mathrm{s}$. & 855 \\
\hline 54, rue Pierre-Audry & 58 & $\mathrm{II}^{\mathrm{e}} \mathrm{s}$. & 1841 \\
\hline Rue de la Favorite & 243 & $1^{\text {re }}$ moitié $1^{\mathrm{er}} \mathrm{s}$. & 301,9 \\
\hline Rue de la Favorite & 396 & $1^{\text {re }}$ moitié $~^{\text {er }} \mathrm{s}$. & 486,8 \\
\hline Rue de la Favorite & 255 & $1^{\text {re }}$ moitié $\mathrm{l}^{\mathrm{er}} \mathrm{s}$. & 509,8 \\
\hline Rue de la Favorite & 114 & $1^{\text {re }}$ moitié $1^{\mathrm{er}} \mathrm{s}$. & 633,9 \\
\hline Rue de la Favorite & 405 & $1^{\text {re }}$ moitié $1^{\text {er }} \mathrm{s}$. & 663,2 \\
\hline Rue de la Favorite & 299 & $2^{\mathrm{e}}$ moitié $\mathrm{I}^{\mathrm{er}} \mathrm{s}$.-début $\mathrm{II}^{\mathrm{e}} \mathrm{s}$. & 37,2 \\
\hline Rue de la Favorite & 80 & $2^{\mathrm{e}}$ moitié $\mathrm{I}^{\mathrm{er}} \mathrm{s}$.-début $\mathrm{II}^{\mathrm{e}} \mathrm{s}$. & 52,8 \\
\hline Rue de la Favorite & 199 & $2^{\mathrm{e}}$ moitié $\mathrm{l}^{\mathrm{er}} \mathrm{s}$--début II $\mathrm{s}$. & 68,9 \\
\hline Rue de la Favorite & 131 & $2^{\mathrm{e}}$ moitié $\mathrm{I}^{\mathrm{er}} \mathrm{s}$-début $\|^{\mathrm{e}} \mathrm{s}$. & 73,2 \\
\hline Rue de la Favorite & 76 & $2^{\mathrm{e}}$ moitié $\mathrm{I}^{\mathrm{er}} \mathrm{s}$.-début $\mathrm{II}^{\mathrm{e}} \mathrm{s}$. & 97,9 \\
\hline Rue de la Favorite & 35 & $2^{\mathrm{e}}$ moitié $\mathrm{I}^{\mathrm{er}} \mathrm{s}$.-début $\mathrm{I}^{\mathrm{e}} \mathrm{s}$. & 105,3 \\
\hline Rue de la Favorite & 281 & $2^{\mathrm{e}}$ moitié $\mathrm{I}^{\mathrm{er}} \mathrm{s}$.-début $\mathrm{II}^{\mathrm{e}} \mathrm{s}$. & 138,6 \\
\hline Rue de la Favorite & 328 & $2^{\mathrm{e}}$ moitié $\mathrm{I}^{\mathrm{er}} \mathrm{s}$.-début $\mathrm{II}^{\mathrm{e}} \mathrm{s}$. & 153,4 \\
\hline Rue de la Favorite & 7 & $2^{\mathrm{e}}$ moitié $\mathrm{I}^{\mathrm{er}} \mathrm{s}$.-début $\mathrm{II}^{\mathrm{e}} \mathrm{s}$. & 153,6 \\
\hline Rue de la Favorite & 98 & $2^{\mathrm{e}}$ moitié $\mathrm{I}^{\mathrm{er}} \mathrm{s}$.-début $\mathrm{II}^{\mathrm{e}} \mathrm{s}$. & 170,3 \\
\hline Rue de la Favorite & 31 & $2^{\mathrm{e}}$ moitié $\mathrm{I}^{\mathrm{er}} \mathrm{s}$.-début $\mathrm{I}^{\mathrm{e}} \mathrm{s}$. & 234,2 \\
\hline Rue de la Favorite & 33 & $2^{\mathrm{e}}$ moitié $\mathrm{I}^{\mathrm{er}} \mathrm{s}$.-début $\mathrm{II}^{\mathrm{e}} \mathrm{s}$. & 278,7 \\
\hline Rue de la Favorite & 233 & $2^{\mathrm{e}}$ moitié $\mathrm{I}^{\mathrm{er}} \mathrm{s}$.-début $\mathrm{II}^{\mathrm{e}} \mathrm{s}$. & 443,8 \\
\hline Rue de la Favorite & 295 & $2^{\mathrm{e}}$ moitié $\mathrm{I}^{\mathrm{er}} \mathrm{s}$.-début $\mathrm{II}^{\mathrm{e}} \mathrm{s}$. & 539,4 \\
\hline Rue de la Favorite & 8 & $2^{\mathrm{e}}$ moitié $\mathrm{I}^{\mathrm{er}} \mathrm{s}$.-début $\mathrm{II}^{\mathrm{e}} \mathrm{s}$. & 544,2 \\
\hline Rue de la Favorite & 132 & $2^{\mathrm{e}}$ moitié $\mathrm{I}^{\mathrm{er}} \mathrm{s}$.-début $\mathrm{II}^{\mathrm{e}} \mathrm{s}$. & 580,6 \\
\hline Rue de la Favorite & 52 & $2^{\mathrm{e}}$ moitié $\mathrm{I}^{\mathrm{er}} \mathrm{s}$.-début $\|^{\mathrm{e}} \mathrm{s}$. & 853,4 \\
\hline Rue de la Favorite & 59 & $1^{\text {re }}$ moitié $\|^{\mathrm{e}} \mathrm{s}$. & 28,5 \\
\hline Rue de la Favorite & 160 & $1^{\text {re }}$ moitié $\|^{\mathrm{e}} \mathrm{s}$. & 66,7 \\
\hline Rue de la Favorite & 231 & $1^{\text {re }}$ moitié $\|^{\mathrm{e}} \mathrm{s}$. & 156,7 \\
\hline Rue de la Favorite & 29 & $1^{\text {re }}$ moitié $\|^{\mathrm{e}} \mathrm{s}$. & 310,2 \\
\hline Rue de la Favorite & 27 & $1^{\text {re }}$ moitié $\|^{\mathrm{e}} \mathrm{s}$. & 377,9 \\
\hline Rue de la Favorite & 53 & $1^{\text {re }}$ moitié $\|^{\mathrm{e}} \mathrm{s}$. & 483,1 \\
\hline Rue de la Favorite & 201 & $1^{\text {re }}$ moitié $\|^{\mathrm{e}} \mathrm{s}$. & 539,9 \\
\hline Rue de la Favorite & 24 & $1^{\text {re }}$ moitié $\|^{\mathrm{e}} \mathrm{s}$. & 594,3 \\
\hline Voie de l'Océan & 251 & 3 premiers quarts $\|^{\mathrm{e}} \mathrm{s}$. & 1254,4 \\
\hline Voie de l'Océan & 235 & 3 premiers quarts $\|^{\mathrm{e}} \mathrm{s}$. & 850 \\
\hline Voie de l'Océan & 152 & $3^{\mathrm{e}}$ quart $\mathrm{II}^{\mathrm{e}}$-début $\mathrm{II}^{\mathrm{e}} \mathrm{s}$. & 283,2 \\
\hline Voie de l'Océan & 153 & $1^{\text {re }}$ moitié $~^{e r} \mathrm{~s}$. & 1250 \\
\hline 62, rue du Commandant-Charcot & 2 & $2^{\mathrm{e}}$ moitié $\mathrm{l}^{\mathrm{er}} \mathrm{s}$. & 190,5 \\
\hline
\end{tabular}

ont reçu un aménagement de couverture (bâtières de tuiles, couverture de tuiles posées à plat, plaques de marbre, pierre dormante d'un broyeur à olives) ; dans 16 cas, des cols d'amphores plantés verticalement au centre des bûchers semblent correspondre à des éléments de signalisation et/ou à des conduits à libation ; des fragments de stèles funéraires ont été découverts en remploi dans un drain proche ; dans un cas (bûcher 45), le bûcher est accolé à un bloc taillé de forme parallélépipédique avec un logement central semblant avoir pu supporter une stèle funéraire (un fragment d'épitaphe, gravé sur une plaque de marbre, a été découvert à proximité) ; enfin quelques dépôts secondaires 
Tabl. XXI - Masses osseuses relevées dans une sélection de bûchers provençaux.

\begin{tabular}{|c|c|c|c|}
\hline Site & $N^{\circ}$ bûcher & $\begin{array}{l}\text { Datation } \\
\text { (apr. J.-C.) }\end{array}$ & $\begin{array}{l}\text { Masse osseuse brûlée } \\
\text { (en g, lots complets) }\end{array}$ \\
\hline Communaux, Vernègues & B-1 & $75-300$ & 193,5 \\
\hline Communaux, Vernègues & B-2 & $150-200$ & 110,4 \\
\hline Communaux, Vernègues & B-3 & $150-200$ & 404 \\
\hline Communaux, Vernègues & B-4 & $100-150$ & 90,5 \\
\hline Communaux, Vernègues & B-5 & $50-150$ & 1774,2 \\
\hline Communaux, Vernègues & B-6 & $150-200$ & 181,2 \\
\hline Communaux, Vernègues & B-8 & $100-200$ & 30,3 \\
\hline Communaux, Vernègues & B-12 & $75-200$ & 392,6 \\
\hline Communaux, Vernègues & B-16 & $150-200$ & 817,6 \\
\hline Communaux, Vernègues & B-17 & $75-200$ & 59,9 \\
\hline Communaux, Vernègues & B-19 & $75-100$ & 32,3 \\
\hline Communaux, Vernègues & B-20 & $175-200$ & 437,5 \\
\hline Communaux, Vernègues & B-21 & $75-300$ & 231,2 \\
\hline Communaux, Vernègues & B-22 & $75-125$ & 234,9 \\
\hline Communaux, Vernègues & B-24 & $75-300$ & 79,3 \\
\hline Communaux, Vernègues & B-25 & $100-150$ & 3,6 \\
\hline Communaux, Vernègues & B-27 & $100-150$ & 109,2 \\
\hline Communaux, Vernègues & B-29 & $150-200$ & 559,8 \\
\hline Communaux, Vernègues & B-30 & $50-150$ & 66,7 \\
\hline Communaux, Vernègues & B-31 & $150-200$ & 397,6 \\
\hline Communaux, Vernègues & B-32 & $75-200$ & 6,1 \\
\hline Communaux, Vernègues & B-33 & $100-150$ & 690,8 \\
\hline Communaux, Vernègues & B-34 & $50-150$ & 153,7 \\
\hline Communaux, Vernègues & B-35 & jer $\mathrm{s}$. & 368 \\
\hline Communaux, Vernègues & B-37 & vers 150 & 570,2 \\
\hline Communaux, Vernègues & B-38 & $100-150$ & 142,4 \\
\hline Communaux, Vernègues & B-39 & $75-150$ & 756,4 \\
\hline Communaux, Vernègues & B-40 & $75-300$ & 442,5 \\
\hline Communaux, Vernègues & B-41 & $100-200$ & 454,5 \\
\hline Communaux, Vernègues & B-44 & $200-300$ & 66 \\
\hline Communaux, Vernègues & B-45 & $75-150$ & 169,8 \\
\hline Communaux, Vernègues & B-48 & $150-200$ & 1042,5 \\
\hline Communaux, Vernègues & B-49 & $75-150$ & 268,6 \\
\hline Communaux, Vernègues & B-50 & $75-200$ & 473,8 \\
\hline Communaux, Vernègues & B-57 & - & - \\
\hline Communaux, Vernègues & B-58 & post 140 & 165,1 \\
\hline Communaux, Vernègues & B-59 & $50-150$ & 5,6 \\
\hline Communaux, Vernègues & B-60 & $50-150$ & 81,8 \\
\hline Communaux, Vernègues & B-61 & $75-300$ & 439,8 \\
\hline Communaux, Vernègues & B-63 & $75-150$ & 12,6 \\
\hline Communaux, Vernègues & B-65 & $200-230$ & 547,7 \\
\hline Communaux, Vernègues & B-66 & - & 23,7 \\
\hline Communaux, Vernègues & B-67 & $175-230$ & 9,9 \\
\hline Communaux, Vernègues & B-68 & $140-200$ & 939,7 \\
\hline Communaux, Vernègues & B-69 & $150-200$ & 341,1 \\
\hline Communaux, Vernègues & B-71 & - & 319,7 \\
\hline L'Héritière, Vernègues & B-209 & $50-100$ & 116,8 \\
\hline Aix-en-Provence & $B-58$ & $150-200$ & 1066,7 \\
\hline Aix-en-Provence & B-48 & $50-75$ & 765 \\
\hline
\end{tabular}


de mobilier, balsamaires et flacons en verre, sont présents. Devons-nous suivre R. Pirling qui choisit d'accorder aux bûchers de Krefeld-Gellep, caractérisés par des déficits importants d'ossements, le statut de sépultures en raison de la présence de mobilier secondaire (Pirling, 2002, p. 505) ?

Les difficultés rencontrées pour interpréter ce type de structure funéraire sont les mêmes dans les contextes romains de Sainte-Barbe à Marseille (Moliner et al., 2003, p. 102-104 ; Richier, 2005, p. 199-209) ; sur les neuf bûchers en fosse complets présents sur le site, cinq contiennent des masses osseuses totales s'échelonnant entre $67 \mathrm{~g}$ et $631 \mathrm{~g}$, avec une moyenne de $336 \mathrm{~g}$ (écart-type : 216). La part prélevée des ossements dans ces bûchers, en comparaison avec le poids moyen théorique d'un sujet complet, peut être estimée en moyenne à $75 \%$ d'un squelette. Quatre de ces fosses ont montré par ailleurs que le prélèvement intéressait principalement le haut du corps (crâne, tronc, membres supérieurs). Ces bûchers sont cependant parfaitement intégrés dans l'espace funéraire et rien ne les distingue des sépultures environnantes ou des bûchers contenant des sujets entiers, interprétés a priori comme des « tombes-bûchers ». Seule une répartition topographique particulière est notable pour quatre de ces bûchers : ils sont rassemblés dans le secteur sud de l'ensemble funéraire (secteur recelant le plus de dépôts secondaires en ossuaire ou en fosse), alors que les bûchers conservant une masse osseuse acceptable sont rassemblés dans le secteur nord. Pour trois de ces bûchers enfin, des dépôts secondaires de mobilier sont présents, signifiant que même si des prélèvements osseux ont eu lieu, un rite a été pratiqué avant le scellement de la fosse. Un autre exemple découvert sur le site inédit récemment fouillé de Croix-Rouge à Orange dans le Vaucluse (étude en cours par R. Gaday et A. Richier), illustre également les égards pris pour une structure interprétée comme un simple bûcher. Il s'agit du bûcher 20, situé, comme trois de ses homologues, à l'extérieur d'un enclos contenant des dépôts de crémation en urne de calcaire et de marbre, en face d'un mausolée. La fosse, aux parois fortement rubéfiées, a des dimensions restreintes ( $1 \mathrm{~m}$ sur $0,60 \mathrm{~m}$ ) mais est beaucoup plus profonde que les autres bûchers. Un bloc de calcaire grossièrement taillé et anépigraphe (large et haut de 0,50 m, épais de 0,20 m) est fiché au centre de la fosse, dépassant du niveau de creusement de 0,35 m. Il s'agit sans conteste d'un marqueur aérien de signalisation. Le dépôt secondaire d'un balsamaire complet et non brûlé en verre est présent à la surface du comblement. Ce dernier scelle la couche de crémation qui contient, outre de nombreux vestiges du mobilier primaire,
426 g d'ossements brûlés, attestant un prélèvement osseux important une fois la crémation achevée.

Le site de Sainte-Barbe montre qu'au sein d'un même ensemble funéraire, durant la même période (milieu du $\mathrm{I}^{\mathrm{er}}$ s.-fin $\mathrm{du} \mathrm{II}^{\mathrm{e}} \mathrm{s}$.) coexistent des pratiques différentes, consistant à prélever une part plus ou moins importante des ossements une fois la crémation achevée. Cette distinction soulève bien sûr des interrogations et conduit à considérer le bûcher dans son intégralité : doit-on interpréter les fosses livrant des masses d'ossements pouvant correspondre à l'intégralité des restes comme des sépultures alors que les autres ne seraient «que » des bûchers individuels ? La présence ou l'absence apparente de prélèvements osseux n'influe pas sur la taille des fosses, ce qui laisse supposer une pratique similaire, que la fosse s'apparente ou non à un sépulcre. En revanche, une différence a été notée pour ce qui concerne l'épaisseur de la couche de crémation : les cinq bûchers montrant des prélèvements osseux renferment une couche de crémation épaisse en moyenne de $0,11 \mathrm{~m}$ tandis que les quatre bûchers contenant des individus complets ont une couche de crémation d'une épaisseur moyenne de $0,18 \mathrm{~m}$. Il semble donc que, lorsque des prélèvements osseux ont lieu, ils concernent également les charbons et résidus de crémation aux alentours.

La situation paraît assez différente en Languedoc, mais le corpus disponible est beaucoup plus restreint (tabl. XXII). Sur 19 bûchers en fosse languedociens complets ou presque, 13 (près des deux tiers) conservent une masse d'os humains brûlés supérieure à $1000 \mathrm{~g}$ et trois autres une masse comprise entre $600 \mathrm{~g}$ et $1000 \mathrm{~g}$. Trois encore seulement livrent moins de $200 \mathrm{~g}$ d'os. La moyenne des masses osseuses est de 1163,7 g et l'écart-type de 558,1. On observe, comme pour l'Auvergne, que la documentation languedocienne comporte une forte proportion de contextes ruraux (environ la moitié), alors que les sites provençaux et rhône-alpins étudiés sont principalement urbains. Il est toutefois impossible en l'état des données de mettre en évidence des différences de pratiques entre les contextes ruraux et urbains ou en fonction de la chronologie des bûchers. La majorité de la documentation méridionale concerne en effet le II $^{\mathrm{e}}$ s. apr. J.-C. : sur 59 bûchers datés avec suffisamment de précision, $62 \%$ appartiennent au $\mathrm{II}^{\mathrm{e}} \mathrm{s}$., $18 \%$ au I ${ }^{\mathrm{er}}$ s. et $20 \%$ sont datés entre 50 et 150 apr. J.-C. Globalement, la valeur moyenne de la masse osseuse ne varie pratiquement pas $\left(666,3 \mathrm{~g}\right.$ au $\mathrm{I}^{\mathrm{er}} \mathrm{s}$. et $558,7 \mathrm{~g}$ au $\mathrm{II}^{\mathrm{e}} \mathrm{s}$.), alors que la variabilité est toujours très élevée (écart-types respectifs de 537 et 511,17$)$.

Les rares bûchers pour lesquels les déficits pondéraux sont mis en évidence montrent que, contrairement à Sainte- 
Tabl. XXII - Masses osseuses relevées dans une sélection de bûchers languedociens.

\begin{tabular}{|l|c|c|c|}
\hline \multicolumn{1}{|c|}{ Site } & N $^{\circ}$ bûcher & $\begin{array}{c}\text { Datation } \\
\text { (apr. J.-C.) }\end{array}$ & $\begin{array}{c}\text { Masse osseuse brûlée } \\
\text { (en g, lots complets) }\end{array}$ \\
\hline 78, avenue Jean-Jaurès, Nîmes & B-1154 & $100-150$ & 132 \\
\hline 78, avenue Jean-Jaurès, Nîmes & B-1004 & Ir-II s. $^{\text {(er }}$ & 173,1 \\
\hline Soumaltre, Aspiran & B-11190 & $20-40$ & 436,5 \\
\hline Peyre Plantade, Clermont-l'Hérault & & $25-70$ & 637,5 \\
\hline Soumaltre, Aspiran & B-11172 & $20-40$ & 788,2 \\
\hline 59, avenue Jean-Jaurès, Nîmes & B-2014 & $100-200$ & 979,4 \\
\hline 59, avenue Jean-Jaurès, Nîmes & B-2031 & $100-200$ & 1005,3 \\
\hline L'Hôtel-Dieu, Narbonne & B-2390 & $50-100$ & 1035,4 \\
\hline Mas de Vignoles IX, Nîmes & B-2249 & $70-200$ & 1102,7 \\
\hline 78, avenue Jean-Jaurès, Nîmes & B-1295 & $125-200$ & 1114 \\
\hline Mas de Vignoles IX, Nîmes & B-2247 & $70-200$ & 1258,4 \\
\hline L'Estel, Vers-Pont-du-Gard & B-11 & $50-250$ & 1393,2 \\
\hline L'Estel, Vers-Pont-du-Gard & B-13 & $?$ & 1494,1 \\
\hline 78, avenue Jean-Jaurès, Nîmes & B-1055 & $75-150$ & 1495,3 \\
\hline La Gallière, Montpellier & B-20001 & $25-75$ & 1551,5 \\
\hline L'Estel, Vers-Pont-du-Gard & B-12 & $?$ & 1677,1 \\
\hline L'Estel, Vers-Pont-du-Gard & B-5 & $70-150$ & 1893,3 \\
\hline L'Estel, Vers-Pont-du-Gard & B-10 & $70-200$ & 1953,2 \\
\hline Magaille, Nîmes & B-1021 & $50-200$ & 1989,4 \\
\hline
\end{tabular}

Barbe, le ramassage ne paraît pas privilégier une région anatomique en particulier, tous les cas de figure étant rencontrés. C'est le cas par exemple sur le site des Communaux de Saint-Cézaire à Vernègues (Chapon et al., 2004, p. 121), ou pour les bûchers languedociens. Toutefois, en RhôneAlpes ou en Auvergne, ainsi qu'en Languedoc, ce travail n'a jamais été entrepris à l'échelle d'un site possédant une grande quantité de bûchers en fosse bien conservés.

Les bûchers de la Brunerie à Voiron (Isère), de Chambussière à Pont-du-Château (Puy-de-Dôme) et de Soumaltre à Aspiran (Hérault), qui conservent l'ossuaire à l'intérieur ou à côté de la fosse de crémation, indiquent qu'une faible masse d'os issus de la couche de crémation n'est pas forcément compensée par celle de l'ossuaire ; le déficit observé sur la somme des deux unités reste encore très élevé : 293,47 g à Voiron, 367,9 g et 565,2 g à Chambussière, et $625,3 \mathrm{~g}$ à Soumaltre. Il en est de même dans le bûcher 30 du Cagalou à Mouriès (Bouches-du-Rhône), dont les résidus ne comportent aucun fragment osseux, tandis que seule une partie ces derniers (157,9 g) est réunie dans l'ossuaire 31 (Marcadal, Paillet, 2003, p. 274 et p. 291).

Il semble donc que la constitution d'un dépôt secondaire de crémation sur le lieu où le corps a brûlé, l'aménagement a posteriori du bûcher par des marqueurs divers évoquant une sépulture comme à Vernègues (stèles, conduits à libation, mobilier), n'impliquent pas une conservation de l'intégralité des restes dans la fosse (voir chapitre VI, p. 317-319).

\section{LES DONNÉES ARCHÉOLOGIQUES}

L'intervention destinée à prélever des ossements se manifeste parfois par un creusement dans la couche charbonneuse. Ces loculi bien marqués doivent être distingués des simples dépressions résultant de l'affaissement différentiel des cendres (effet de paroi). Les sites du Valladas (Bel et al., 2002, p. 94), d'Aspiran (Thernot et al., 2004, p. 319) et du Replat à Aime, livrent plusieurs exemples de ce type : la couche charbonneuse est nettement recreusée parfois jusqu'au fond de la fosse et le comblement de cette fosse se distingue de la couche de résidus par une plus faible proportion de charbons et d'artefacts (fig. 107).

Un autre type de remaniement est illustré par la couche de crémation d'un bûcher daté du troisième quart du $\mathrm{I}^{\mathrm{er}} \mathrm{s}$, fouillé à la Raude à Tassin-la-Demi-Lune, dans la banlieue ouest de Lyon. Le fond du bûcher possède deux dépressions situées de part et d'autre d'une bande de terre en place rubéfiée, comblées de résidus de crémation (fig. 82). L'étude a montré que les artefacts et les ossements se répartissaient de manière différente dans chacune de ces 


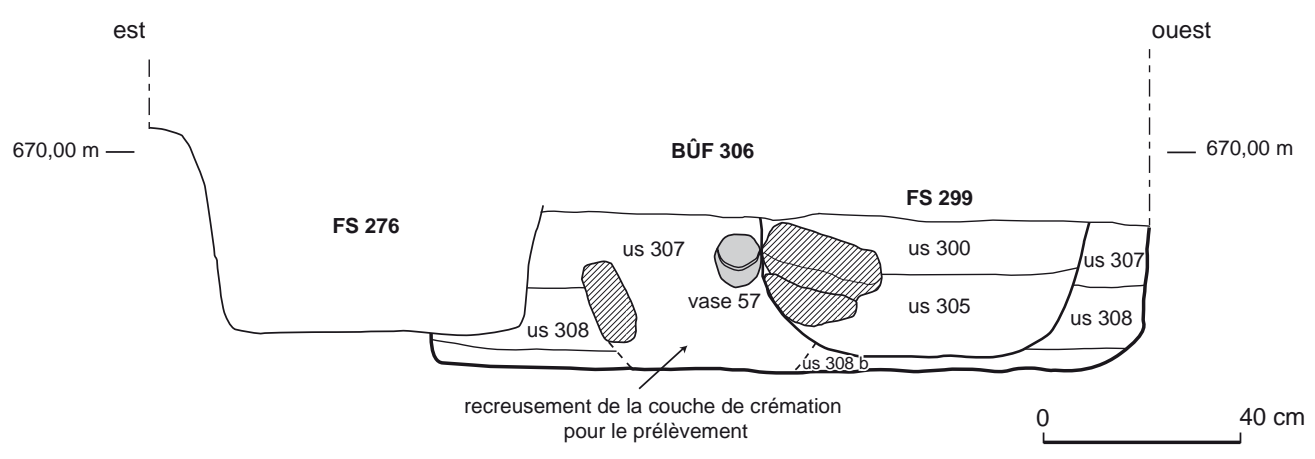

Fig. 107 - Recreusement dans la couche de crémation du bûcher 306

du Replat à Aime, Savoie (relevé : F. Blaizot, Inrap; DAO : V. Bastard, Inrap).

dépressions. En effet, la masse osseuse la plus importante se situe dans la cuvette ouest, tandis que le mobilier calciné est principalement concentré dans la cuvette orientale (fig. 108). En outre, dans cette dernière, le mobilier était concentré en un amas dense placé au sommet de la couche. Deux hypothèses peuvent être envisagées. La première est que le mobilier se trouvait effectivement du côté est sur le bûcher, tandis que l'on aurait brassé le corps en le rabattant du côté ouest, comme à Voiron. La seconde est que la répartition bipartite serait due à un réaménagement a posteriori des vestiges brûlés. Les meilleurs arguments de cette dernière sont apportés par la faible épaisseur de la couche de crémation, surtout du côté oriental, par l'absence de stratigraphie différenciée au sein de cette couche et par le déficit probable ${ }^{12}$ de la masse osseuse, témoignant d'un remaniement complet de la couche. Il apparaît donc fort plausible que les résidus du bûcher aient été sortis de la fosse de manière à être triés pour séparer les restes osseux du mobilier. Cette situation rappelle celle observée à la Favorite dans le bûcher 33 ; de nombreux fragments de céramique recollant avec d'autres issus des résidus restés dans le fond de la fosse ont été retrouvés dans le niveau supérieur de la fosse au-dessus de la couche charbonneuse (Tranoy, 1995b, p. 697). Là aussi, ces faits impliquent que dans un premier temps, la fosse du bûcher a été en partie vidée, puis que le mobilier primaire fut rejeté au moment du rebouchage.

Ce geste est encore illustré par le bûcher 228 du Valladas (Bel et al., 2002, p. 111 et p. 344) qui livre une grande

12. «Probable » car le bûcher contient 338,1 g d'os qui représentent les restes d'un sujet décédé après 2 ans et avant 9 ans ; Warren et Maples (1997, tabl. 2, p. 420-421) donnent une masse de $259 \mathrm{~g}$ pour un enfant de 2 ans et de $636 \mathrm{~g}$ pour un enfant de 4 ans ; Trotter et Hixon (1974, tabl. 3, p. 8), une masse moyenne de $268,4 \mathrm{~g}$ ou $315,8 \mathrm{~g}$ pour un sujet décédé entre 0 et 3 ans et une masse moyenne de 9326 g et 1 132,4 g pour un décès survenu entre 3 et 13 ans. quantité de fragments de vases brûlés, dispersés dans la couche supérieure du comblement de la fosse et sur le sol, autour et au-dessus de la structure. Ce phénomène est d'ailleurs relevé dans plusieurs autres bûchers de ce site, où de manière générale, les couches de remblaiement des bûchers sont le plus souvent mêlées à des résidus charbonneux (Bel et al., 2002, p. 93). Il en est de même dans la fosse 1154 du 78 avenue Jean-Jaurès à Nîmes ; de nombreux éléments de mobilier brûlé (mais très peu d'os), issus des résidus de la crémation, sont mêlés au comblement. La fosse F33 de la rue Sergent- Berthet à Lyon est aussi caractéristique de ces remaniements : le comblement renferme des nodules de terre rubéfiée, des os brûlés, des fragments de vaisselle non brûlée et de nombreux fragments de céramique brûlés appartenant à des vases du dépôt primaire (Tranoy, 1995d, p. 171-172). Ces divers exemples impliquent qu'une partie des résidus prélevés a été réintroduite dans la fosse, avec les terres de remblaiement, probablement après l'opération de tri et de prélèvement des restes osseux attestée par la faible masse d'os conservée.

Il ressort de ces exemples que les bûchers peuvent être profondément remaniés par la nécessité de récupérer des os pour pratiquer la tombe ${ }^{13}$; dans ce cas, il arrive que, pour faciliter le tri, les résidus soient sortis de la fosse, pour être rejetés dans un second temps. Ce procédé n'est toutefois pas systématique, puisque dans certains cas, les résidus paraissent ne pas avoir été déplacés. Le bûcher de Voiron, dans lequel l'organisation du mobilier est respectée, en est un bon exemple. Dans le bûcher 332 de

13. Que ce soit par le mode crématoire ou a posteriori. Des exemples comme celui du bûcher 5 des Oblets à Thérouanne dans le Pas-deCalais (Bura, 2001, p. 174), où la masse osseuse est de 1387 g et les éléments répartis dans la fosse selon la logique anatomique, sont exceptionnels. 


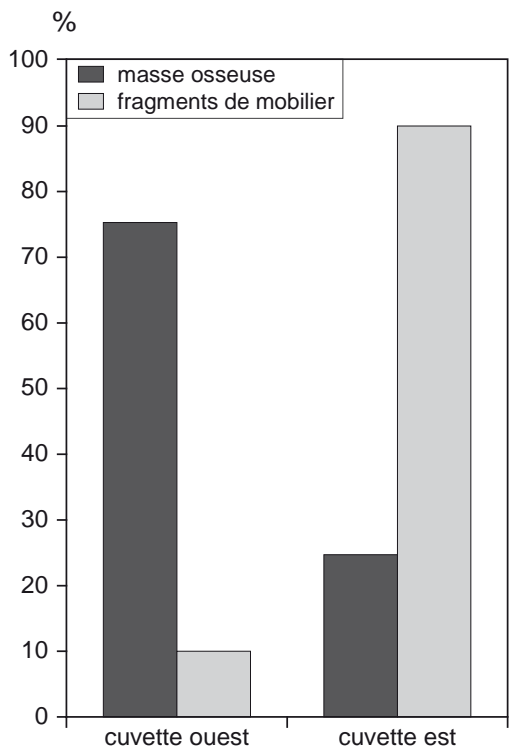

Fig. 108 - Répartition de la masse osseuse dans le bûcher remanié de la Raude à Tassin-la-Demi-Lune, Rhône (DAO : F. Blaizot, d'après les données de J.-L. Gisclon, Inrap).

la Favorite, L. Tranoy remarque que les résidus ont été remués : la majorité des fragments de vases provient de la partie ouest de la fosse alors que certains, appartenant aux mêmes vases, sont issus du centre et de l'ouest, comme si les résidus avaient été déplacés d'est en ouest (Tranoy, 1995b, p. 682). Au 9 rue du Docteur-Horand, à Lyon, C. Bellon note que dans la partie ouest du bûcher, les remontages des vases sont très nombreux de bas en haut de la couche de résidus ; l'auteur attribue ce phénomène à l'effondrement du bûcher, mais sa localisation à une extrémité de la fosse nous conduit à envisager qu'il puisse s'agir des conséquences d'un prélèvement sur place sans extraire la masse des résidus.

L'exemple d'un bûcher fouillé à Chartres, au lieu-dit Pôle Synéo, illustre un mode différent de prélèvement. En effet, au sommet de la couche de crémation, se trouvent des pierres exemptes de traces du feu, manifestement déposées une fois l'ustion terminée (fig. 109). Un ensemble de ces pierres recouvrent des esquilles osseuses, indiquant que le bûcher n'a pas été nettoyé préalablement à leur dépôt. Ces pierres ont des dimensions variables, mais un lot formé des plus petites occupe une surface équivalente $\mathrm{au}$ bloc le plus important. Les fouilleurs ont émis l'hypothèse qu'elles puissent correspondre à l'installation d'une planche destinée à faciliter le prélèvement des os dans le bûcher, sachant que celui-ci mesurait 4,34 m de longueur, pour une largeur minimale de 2,58 $\mathrm{m}$. Compte tenu de la

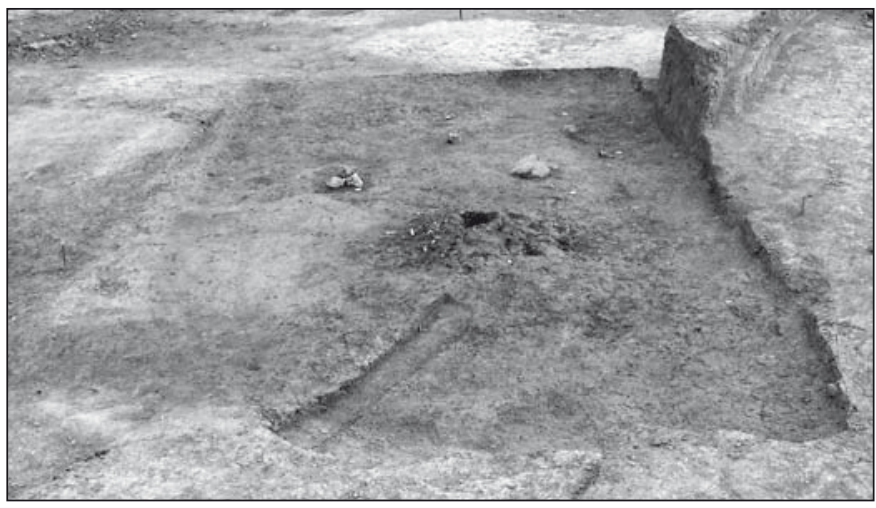

Fig. 109 - Aménagement de blocs sur la couche de crémation du bûcher du Pôle Synéo à Chartres, Eure-et-Loir (cliché : P. Georges, Inrap).

profondeur de la fosse (au moins 0,50 m), si l'opérateur s'était tenu sur une planche en appui sur les bords, il se serait trouvé dans une situation inconfortable, trop haut au-dessus des restes. Ainsi, le dispositif envisagé à l'intérieur de la fosse permettait de traiter les vestiges (nettoyage et/ ou ramassage et/ou tri), à une hauteur raisonnable et sans avoir à poser les pieds sur la couche de crémation, soit parce que l'on ne voulait pas porter atteinte aux restes, soit plus probablement parce que celle-ci était encore incandescente, le refroidissement nécessitant plus de 12 heures (Lambot, 1994 ; Grévin, 2005). La disposition des pierres indique que la planche, dont les appuis sont distants de 0,70 m, était placée presque au centre de la fosse, perpendiculaire à son axe longitudinal. Adoptant une situation centrale, l'opérateur a donc eu vraisemblablement ainsi accès à l'ensemble de la fosse, qui au final conservait 1 161,9 $\mathrm{g}$ d'ossements humains sur lesquels 331,1 g se trouvaient dans le vase ossuaire posé dans le bûcher.

\section{LES RÉAGENCEMENTS DE LA COUCHE DE RÉSIDUS DE CRÉMATION}

Dans certains bûchers toutefois, les résidus sont réagencés de manière raisonnée, laissant entendre que leur présence dans le bûcher signifie bien plus que l'abandon sur place des vestiges de la crémation. Ce geste est clairement illustré par l'exemple du bûcher 3060 de Peyre Plantade à Clermont-l'Hérault daté des deuxième et troisième quarts du $\mathrm{I}^{\mathrm{er}} \mathrm{s}$. apr. J.-C. Les résidus de crémation sont conservés sur le fond de la fosse sous forme de lambeaux et de lentilles éparses, sauf dans la partie nord où ils forment un amas quadrangulaire de $0,40 \mathrm{~m}$ à $0,50 \mathrm{~m}$ 
de largeur à la base, de 1,60 m de longueur et de 0,16 m d'épaisseur, recouvert par une série de tegulae disposées en bâtière. Si les lambeaux peuvent correspondre à des vestiges en place de la couche de crémation, l'accumulation des résidus charbonneux en un amas semble correspondre à un regroupement intentionnel de la plus grande partie des résidus du bûcher; la forme définie par les résidus et la présence des tegulae suggèrent un coffrage. La répartition des pièces osseuses brûlées, fondée sur les indices pondéraux, montre une dispersion importante des restes humains dans la fosse, avec toutefois une concentration des éléments dans le secteur situé à l'est et dans le prolongement (et à l'extérieur) de l'amas coffré (fig. 110). La présence de fragments à distance de cet amas, et de part et d'autre de celui-ci, témoigne de l'éparpillement des pièces avant le regroupement des résidus de la crémation, ce qui tendrait à montrer que l'amas ne résulte pas de la conduite de la crémation, mais d'un aménagement terminal. Par ailleurs, on observe que la répartition des restes osseux est globalement cohérente d'un point de vue anatomique si l'on met à part la dispersion des éléments de membres inférieurs, phénomène fréquent et lié au comportement particulier des membres inférieurs pendant la crémation (Blaizot, 2005, p. 26), ou à la conduite de la crémation (voir chapitre II, p. 147-150). On est donc en présence d'une répartition "normale " pour la crémation d'un corps placé sur le dos, tête à l'est et pieds à l'ouest. Le respect de la logique anatomique suppose que le regroupement des restes de la crémation s'est effectué selon un axe approximativement perpendiculaire à celui du corps de l'individu. Dans le cas contraire, la répartition des différentes régions anatomiques serait aléatoire. Des empreintes de sept tuiles imprimées à la surface de l'amas et quelques fragments de tegulae retrouvés en place permettent de restituer un coffre quadrangulaire d'environ $0,50 \mathrm{~m}$ de largeur à la base et de 1,75 m de longueur, constitué par deux rangs de quatre tegulae disposées en bâtière. Des fragments d'imbrices retrouvés dans le comblement au-dessus de l'amas invitent à restituer sur la bâtière un rang de tuiles couvre-joints complètement détruites par les labours. Dans ce cas, l'aménagement de la bâtière montre que l'amas de forme oblongue, constitué à la fin de la crémation, avait bien pour fonction de matérialiser de manière mimétique et symbolique la présence du sujet brûlé dans la fosse. Pourtant la masse totale d'os conservée (637,5 g) apparaît inférieure à la valeur minimale attendue pour le squelette calciné d'un sujet adulte (tabl. XVIII, p. 152). Le déficit est trop important pour envisager que les labours aient fait disparaître une partie des restes dans une telle proportion, d'autant plus que la surface de l'amas semble intacte (vestiges et empreintes de tuiles).

Des dispositifs de tuiles en bâtière, installés sur les résidus de crémation dans un bûcher en fosse trouvent des parallèles sur le site de Saint-Lambert à Fréjus (Béraud et al., 1985, p. 13), sur celui des Communaux de Saint-Cézaire (Chapon et al., 2004, p. 171 et p. 193, fosses 40 et 69) ainsi qu'à Narbonne, rue d'Artois, dans le quartier Razimbaud (Dellong, 2002, p. 333). Pour tous ces exemples, il n'est pas précisé si les résidus ont été rassemblés avant d'être recouverts par le dispositif de couverture, ce qui semble être le cas, en revanche, de l'exemplaire de Ruscino à Perpignan (Pyrénées-Orientales) : R. Marichal le décrit comme une fosse rectangulaire recevant le bûcher, dont les cendres ont été rassemblées sous des tegulae disposées en bâtière. D'après le fouilleur, les ossements sont en connexion anatomique ou, du moins, présentent une position qui exclut un déplacement entre le lieu de crémation et le lieu d'inhumation. Le fond de la fosse est en outre fortement rubéfié (Marichal, 1998, p. 125).

Le regroupement des résidus en amas a également été observé sur le site du 78 avenue Jean-Jaurès à Nîmes dans deux bûchers (1055 et 1295), respectivement datés entre 75 et 150 et entre 125 et 200 . Il se traduit dans les deux cas, par un bombement de la couche de résidus de crémation, localisée près de l'une des extrémités de la fosse. Seul le bûcher 1055 a fait l'objet d'une fouille et d'un prélèvement par secteur. Ces données n'ont pas encore été entièrement exploitées ; néanmoins, la répartition des masses osseuses montre une concentration des ossements brûlés dans la zone sud où la couche apparaît légèrement plus épaisse, et regroupe $50 \%$ de la masse osseuse totale. Une observation rapide des restes indique que ce secteur rassemble par ailleurs toutes les régions anatomiques. En revanche, les vestiges de mobilier brûlés semblent répartis de manière à peu près égale dans l'ensemble de la fosse, avec les éléments d'un même vase qui paraissent assez peu dispersés. Cette répartition différentielle fait penser qu'une partie des restes osseux a été regroupée dans la partie sud de la fosse pendant la crémation et non a posteriori. Un coffrage de dalles a été ensuite construit sur le petit amas ainsi constitué, maintenu en place par un calage de dalles et de blocs et sans doute initialement doté d'une couverture. Ce coffrage abrite un dépôt de vases non brûlés (deux cruches en céramique, un gobelet et un balsamaire en verre). Dans la fosse 1295, la protection du petit amas de résidus dans la partie nord-est de la couche charbonneuse 


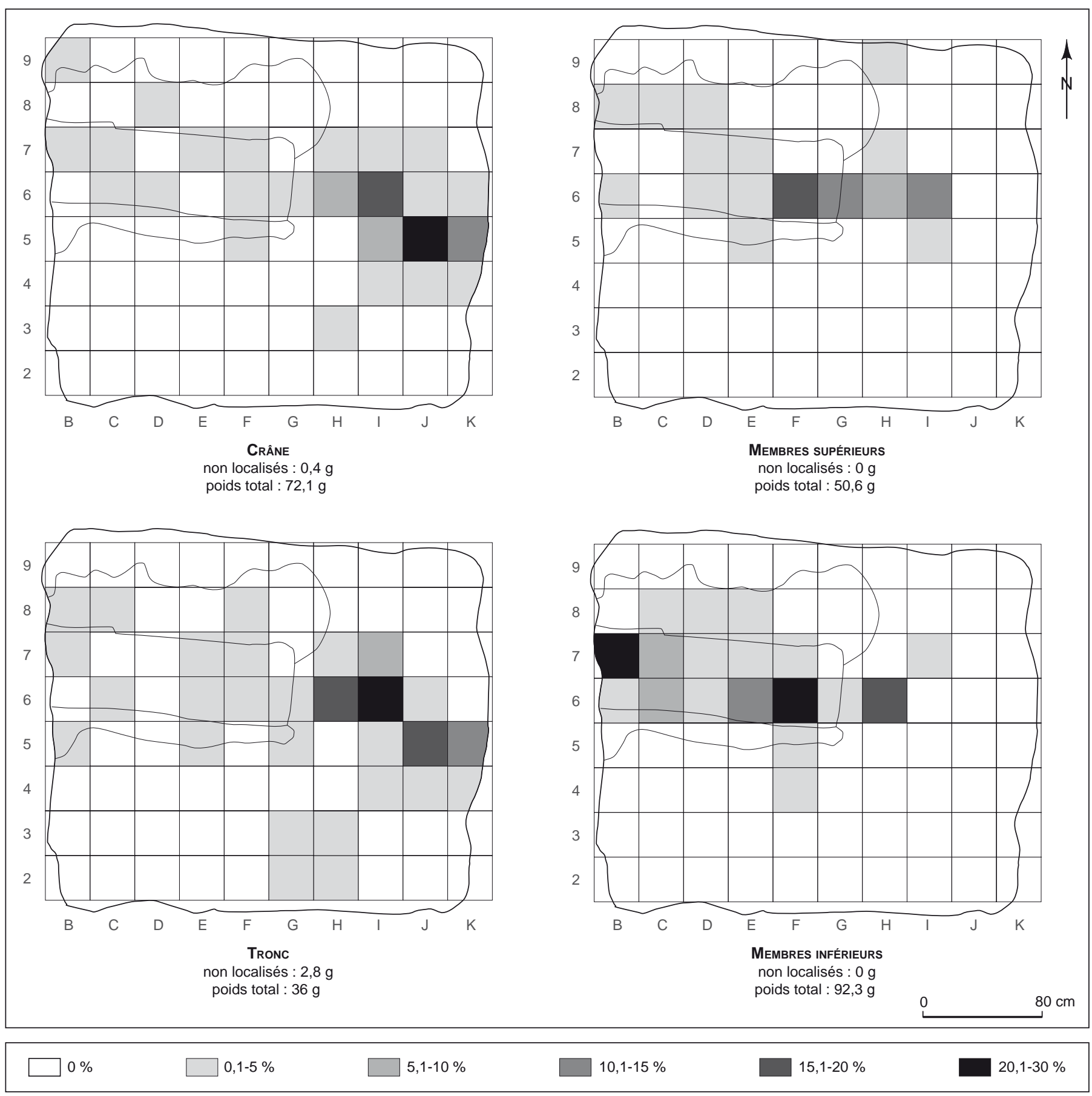

Fig. 110 - Répartition spatiale des restes osseux par régions anatomiques dans le bûcher 3060 de Peyre Plantade à Clermont-l'Hérault, Hérault (relevé : V. Bel, Inrap ; DAO : A. Recolin, Inrap).

est assurée par un simple fragment d'amphore. Celui-ci est associé à un balsamaire en verre non brûlé. La masse d'os brûlés conservée se situe, dans les deux cas, dans la fourchette de variation des valeurs de référence (1495,5 g et $1114 \mathrm{~g}$ ). Dans le bûcher 2247 du Mas de Vignoles IX à
Nîmes (70-200 apr. J.-C.), l'hypothèse d'un regroupement est suggérée par l'organisation de la partie superficielle de la couche de résidus constituée d'ossements brûlés associés à quelques fragments de céramique non brûlés, des petits fragments de charbons de bois et de nombreux nodules 

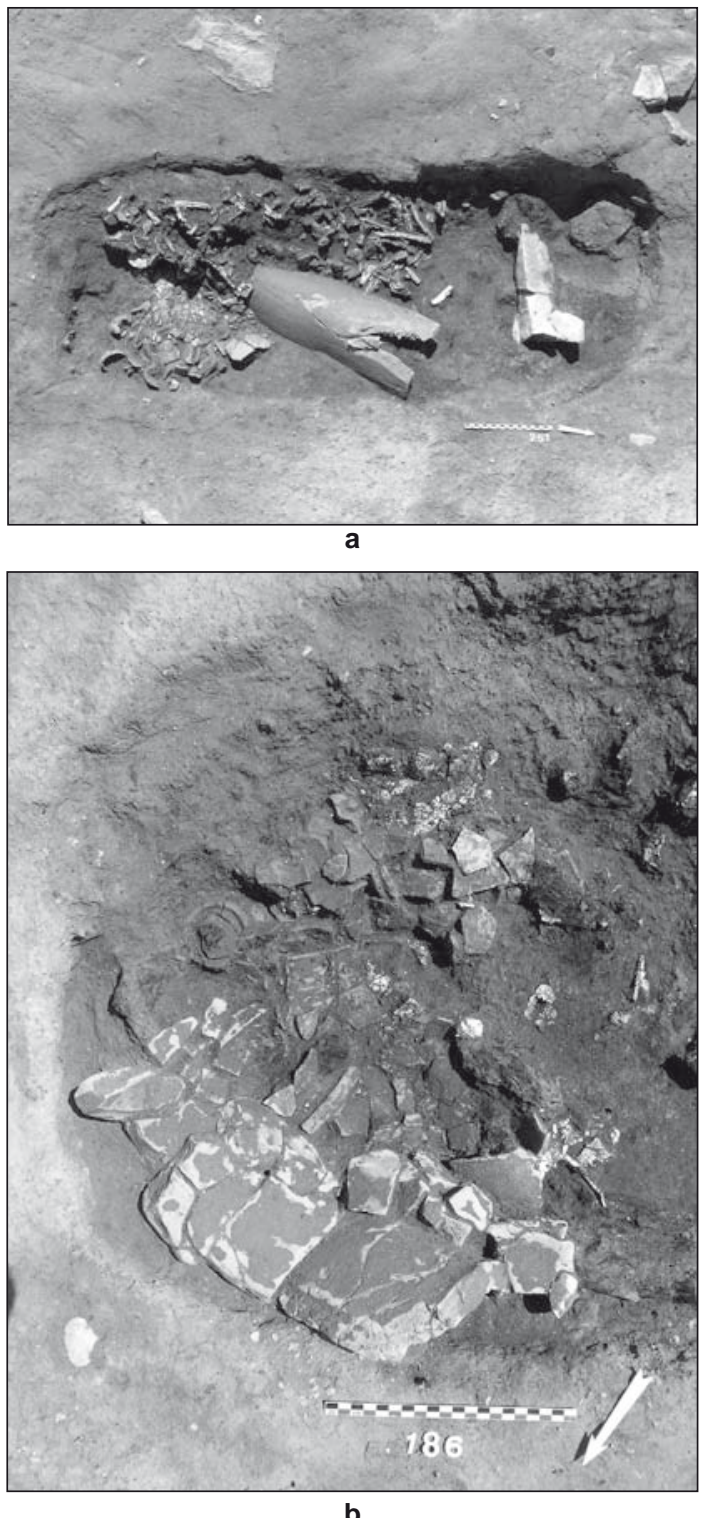

b

Fig. 111 - Effets de parois sur la couche d'os brûlés dans le bûcher 2247 du Mas de Vignoles IX à Nîmes, Gard (cliché : V. Bel, Inrap).

de terre rubéfiée. Là encore, la masse d'os conservée se situe à l'intérieur de la fourchette des valeurs de référence (1258,4 g). Cette couche d'os n'occupe qu'une partie de la fosse et présente des effets de délimitation linéaire qui incitent à restituer les parois d'un contenant en matériau périssable de type coffrage de forme trapézoïdale, plus étroit au sud qu'au nord (0,70 m environ sur 0,15-0,35 m) (fig. 111). Le regroupement n'a pas totalement occulté l'organisation initiale du bûcher dans la mesure où la répartition spatiale des restes osseux est demeurée globalement cohérente du point de vue anatomique. Par ailleurs, les

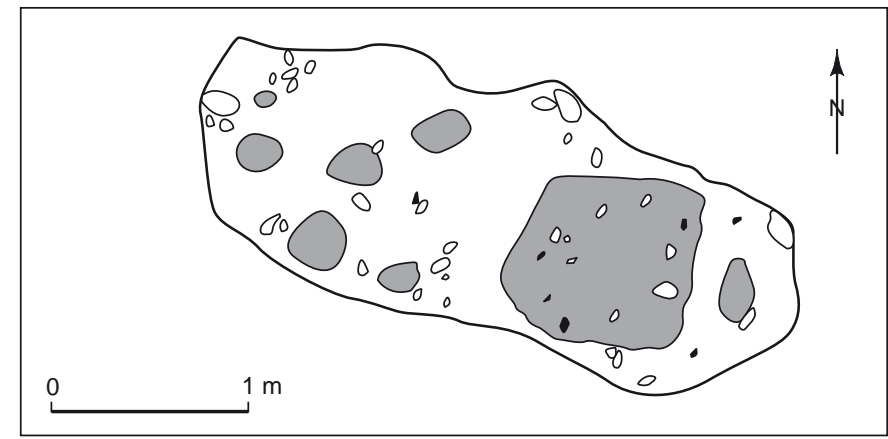

résidus charbonneux

- céramique/verre

galet

Fig. 112 - Réaménagement de la couche de résidus du bûcher 23 du 19 rue des Granges à Lyon (relevé : J. Rouquet, Inrap ; DAO : G. Macabéo, Inrap).

remaniements ont très peu concerné les deux cruches non brûlées et brisées, placées à une extrémité de la fosse sur les résidus de crémation. La présence, parmi les restes osseux, de quelques fragments appartenant à ces deux récipients, incite à penser que l'amas et le coffrage ont cependant été constitués après le dépôt des cruches.

Lorsqu'ils ne nécessitent pas la mise en œuvre de matériaux exogènes ou de remaniements de la structure initiale, ces réaménagements restent difficiles à percevoir. Ainsi, il est très possible que beaucoup n'aient pas été identifiés par les fouilleurs, ce qui expliquerait, par exemple, leur rareté en Rhône-Alpes où les bûchers sont pourtant très nombreux.

L'exemple le plus flagrant d'un réaménagement est celui du bûcher 113 de la Favorite, daté de la première moitié du $\mathrm{II}^{\mathrm{e}}$ s., où les résidus sont amassés autour d'un vase ossuaire, placé dans une petite fosse creusée sur le fond (Tranoy, 1995b, p. 700 ; Blaizot, Tranoy, 2004, fig. 1, p. 173). Le même geste a été relevé dans le bûcher du site de Pôle Synéo à Chartres. Mais ces cas sont particuliers puisqu'il s'agit clairement de la conversion d'une structure primaire en structure secondaire (voir chapitre IV, p. 183-186). Cependant, à Chartres, les résidus ne comportaient que les ossements, ce qui implique une sélection a posteriori illustrant la volonté de ne conserver que les os, à moins que le bûcher ait été dépourvu de mobilier.

Au 19 rue des Granges, à Lyon, le bûcher 23 situé dans le secteur des structures de crémation de la seconde moitié du $\mathrm{I}^{\mathrm{er}} \mathrm{s}$. paraît avoir été en partie raclé ; dans la moitié orientale de la fosse, d'une longueur de 1,60 m, se trouvait en effet rassemblés les résidus de crémation sur une surface globalement quadrangulaire de 0,45 m sur 0,40 m (fig. 112). 
À Vernègues, vingt-trois fosses présentent une concentration de la masse osseuse dans les résidus de la partie centrale du bûcher, dont la répartition révèle des incohérences anatomiques (Chapon et al., 2004, p. 122). L'importance des remaniements a incité les auteurs à les interpréter comme le résultat d'un rassemblement des restes après la crémation plutôt qu'au tisonnage du bûcher et à la conduite de la crémation.

Ces gestes sont remarquablement bien illustrés sur le site de Krefeld-Gellep : les résidus de certains bûchers sont réunis de manière à former une bande au centre de la fosse, à la surface de laquelle se trouvent parfois des dépôts secondaires de mobilier (Pirling, 2002, p. 498-501). Dans le bûcher 5126, les résidus ainsi rassemblés sont couverts de tegulae (Pirling, 2002, p. 496 et fig. 5, p. 497), à l'image des exemples languedociens.

\section{LES PRATIQUES RITUELLES DANS LES BÛCHERS APRÈS LA CRÉMATION}

Comme à Krefeld-Gellep, il existe de nombreux exemples de vases ou d'objets non brûlés déposés sur les résidus charbonneux dans les bûchers en fosse de notre corpus ${ }^{14}$. La présence de mobilier non brûlé dans les bûchers dépourvus a priori d'ossuaire, est assez fréquente et concerne des catégories très diverses. L'une d'elles apparaît plus spécifiquement associée aux structures de crémation : il s'agit des cruches ou des amphores ayant fait l'objet d'un bris intentionnel.

\section{LES BRIS DE CRUCHES ET D'AMPHORES SUR LE BÛCHER}

Dans plusieurs bûchers, des restes de cruches ou d'amphores brisées et non brûlées sont relevés sur la couche de crémation. Le mode de répartition des fragments montre que le bris n'a pu se produire après le dépôt dans la fosse, sous le seul poids des sédiments, mais qu'il a été effectué avant ou au moment de la mise en place dans la fosse.

Ce geste est illustré par le bûcher de Gallière à Montpellier (fig. 113). Sur la couche charbonneuse, est relevé un épandage de fragments appartenant à une amphore conservée à $50 \%$, de type Dressel 20b de Bétique. L'ensemble des indices en présence (dispersion des fragments d'amphore sur environ

14. Certains dépôts sont clairement associés à un ossuaire placé sur le lieu même de la crémation ; ils représentent un cas de figure qui sera discuté dans le chapitre suivant consacré aux structures secondaires. les deux tiers de la surface de la structure, pendage des tessons, limite nette de leur expansion dans la moitié occidentale de la structure, traces de chauffe), montre que le bris et le dépôt de ses tessons ont été effectués directement sur le sommet de la couche de résidus de crémation et contre le bord ouest de la fosse. Une grande partie du bas de panse ne présente pas de traces de chauffe, le col est plus ou moins intact et porte des traces de feu localisées sur la face qui se trouvait au contact des résidus. Il ne s'agit d'ailleurs pas de coups de feu intenses, mais plutôt de légères traces de chauffe. Les tessons qui constituent le fond de l'amphore sont localisés le long et en appui contre la paroi ouest de la fosse, entassés les uns sur les autres. Une trace d'enlèvement ancienne (concrétion sur la face interne) est observée sur un des tessons du milieu de la panse ; elle semble avoir été provoquée par un impact effectué de l'extérieur vers l'intérieur de l'amphore et pourrait résulter d'un geste de bris intentionnel. Le bris de l'amphore a probablement dû se produire par l'éclatement du fond, entraînant ainsi la dispersion des fragments du nord vers le sud. La répartition dans le bûcher des fragments d'amphore correspond à une zone dépourvue d'ossements brûlés (voir supra, p. 124 et fig. 95, p. 125) et très pauvre en mobilier céramique, à l'exception des fragments d'une marmite, dispersés en limite des éléments d'amphore.

Le Languedoc fournit d'autres exemples de cruches brisées sur les résidus après la crémation, comme le bûcher 2247 du Mas de Vignoles IX à Nîmes, déjà mentionné, où deux cruches ont été brisées avant de regrouper une partie des résidus de la crémation. Sur le même site, le bûcher 2249 comporte également les restes de deux cruches brisées au sommet de la couche de résidus à une extrémité de la fosse. Le mode de répartition des tessons montre que le bris des récipients a été suivi d'une dispersion des fragments. Par ailleurs, des traces très discrètes sur la surface de quelques fragments suggèrent moins une exposition à la chaleur du bûcher (dépôt primaire) qu'un dépôt postérieur à la crémation sur des cendres encore chaudes. À Lattes, les fragments non brûlés d'une amphore se trouvent au sommet des résidus de crémation du bûcher 4098 (fig. 114). Certains éléments recouvrent une lampe à huile non brûlée (dépôt secondaire), d'autres s'avèrent partiellement surmontés de résidus ou de fragments d'autres vases brûlés. Le mode de répartition des fragments et les traces de piquetage horizontal observées sur des fragments de panse plaident en faveur d'un bris intentionnel du récipient avant son dépôt. Celui-ci est intervenu après la mise en place du dépôt secondaire de la lampe. 

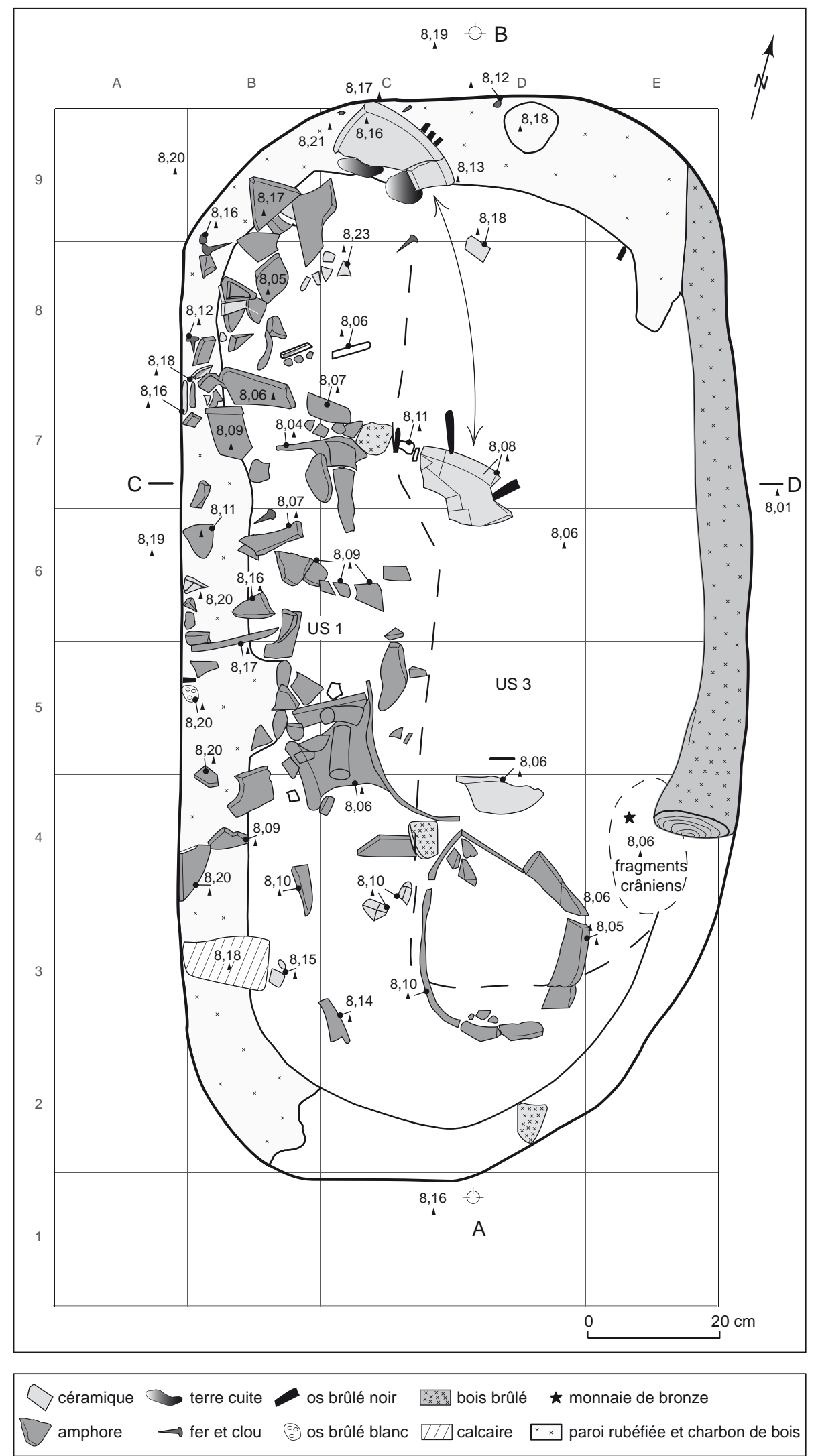

Fig. 113 - Mobilier brisé sur la couche de résidus de crémation

du bûcher de Gallière à Montpellier, Hérault (DAO : R. Lisfranc, Inrap et G. Isnard, Inrap). 


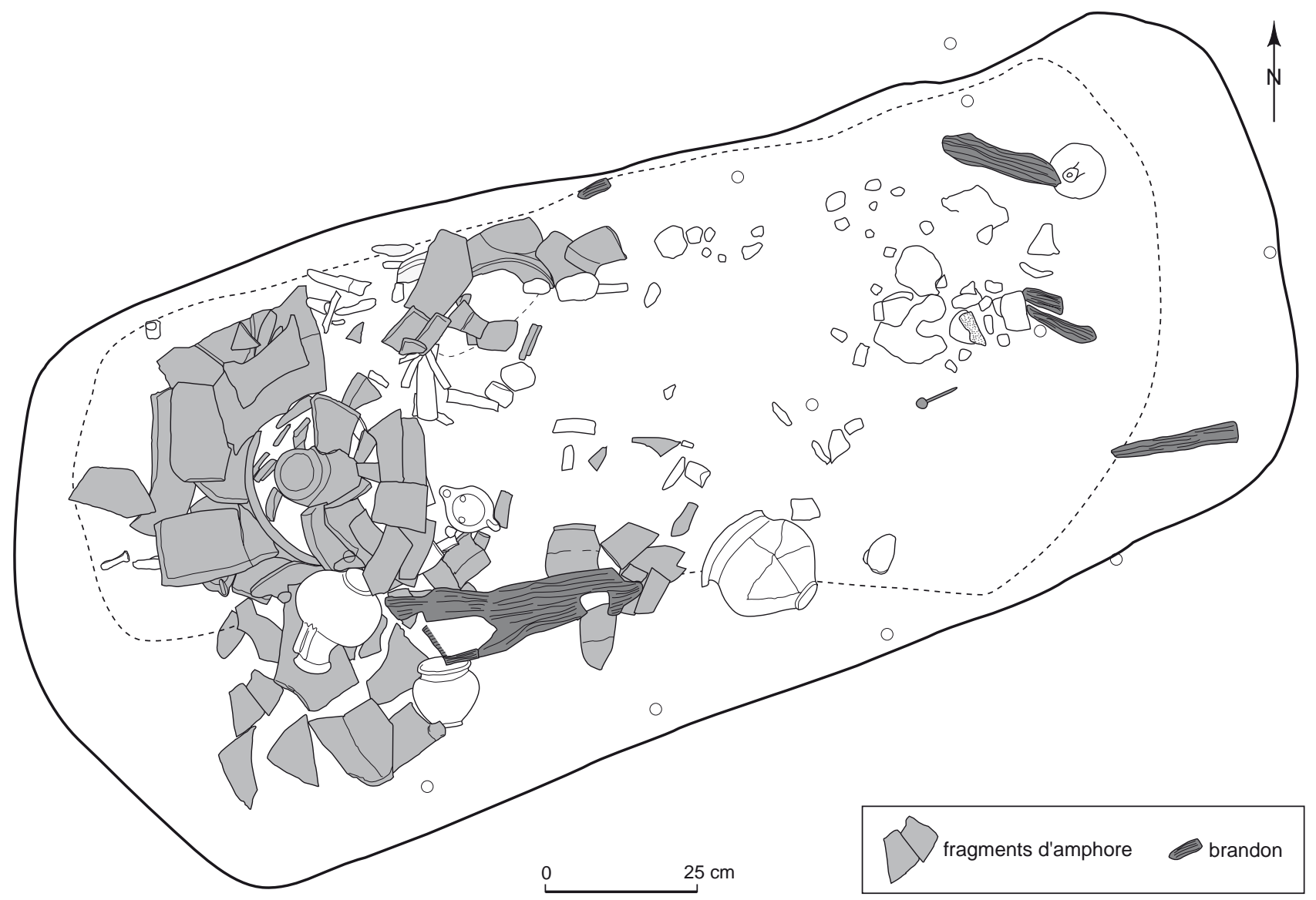

Fig. 114 - Bris de cruche en surface du bûcher 4098 de la Céreirède à Lattes, Hérault (relevé : F. Blaizot, Inrap ; DAO : G. Macabéo, Inrap).

En Rhône-Alpes, plusieurs exemples sont attestés, dont les plus spectaculaires concernent deux bûchers à SaintFréjus à Marennes et celui du 54 rue Pierre-Audry. Dans le bûcher 6318 de Saint-Fréjus, les fragments d'une amphore Gauloise complète apparaissent à la surface de la couche charbonneuse (fig. 115). L'étude détaillée montre que les éléments se répartissent en deux ensembles regroupant des fragments connexes entre eux qui correspondent probablement au bris sur place, avec un déplacement limité des éclats, de morceaux de grandes dimensions. L'un de ces ensembles comprend le col de l'amphore et la partie supérieure du vase (fig. 115, A), et le deuxième (fig. 115, B) le fond et la partie inférieure du vase restés en bon rapport entre eux. Les collages entre ces deux groupes concernent des éléments distants de $0,10 \mathrm{~m}$ à $0,30 \mathrm{~m}$ et sont disposés de manière divergente. Il y a donc eu bris sur place de gros fragments, avec une dispersion d'esquilles, qui fait penser à un choc et à un dépôt par projection. Cette hypothèse est confirmée par la présence d'un point d'impact d'où rayonnent des lignes de fractures sur des fragments de la partie inférieure de la panse. La position des pièces suppose également un dépôt séparé, le fond ayant probablement été jeté avant le col. Un troisième ensemble (fig. 115, C et D) comprend essentiellement des éléments disjoints, éloignés des fragments avec lesquels ils recollent. Ces éléments semblent donc avoir été jetés dans la fosse indépendamment des deux autres lots. L'ensemble de ces observations implique que l'amphore a été brisée hors de la tombe et jetée par morceaux sur la couche charbonneuse depuis le bord, d'abord le fond, puis le col et les fragments restants. Deux grandes cruches du bûcher voisin 6200 ont fait l'objet d'un traitement tout à fait similaire.

Dans le bûcher 58 du 54 rue Pierre-Audry, il s'agit d'une amphore Gauloise incomplète, non brûlée, dont sept grands fragments sont répartis en surface de la couche de résidus. De nombreux tessons lui appartenant ont été recueillis dans la couche de comblement supérieur, ce qui signifie qu'elle a été brisée à l'extérieur de la fosse, avant 


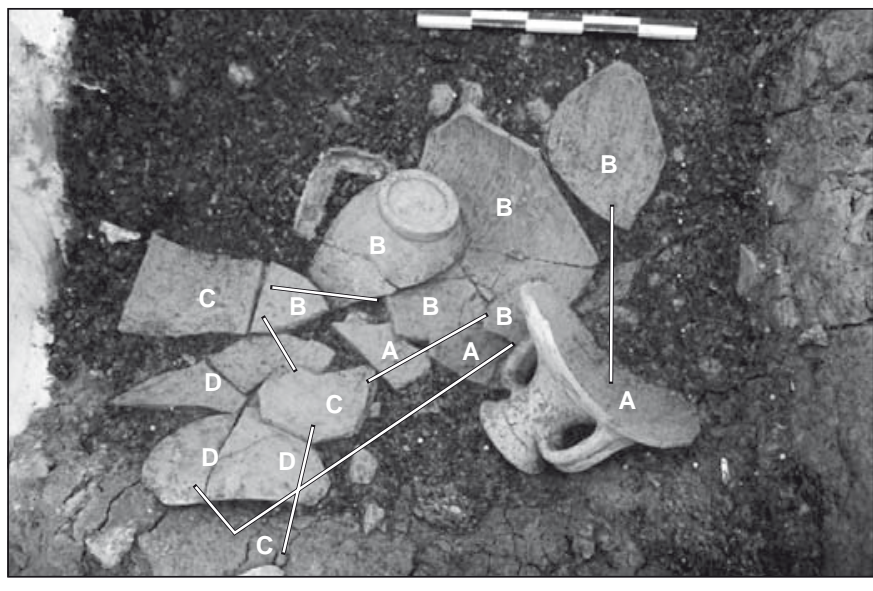

principales liaisons entre les fragments

Fig. 115 - Cruche brisée au sommet du bûcher 6318 de Saint-Fréjus à Marennes, Rhône (cliché : V. Savino, Inrap).

d'être rejetée en partie sur les résidus. En Auvergne, un bûcher du $\mathrm{II}^{\mathrm{e}} \mathrm{s}$. fouillé à la Grande Borne, sur la commune de Clermont-Ferrand, contient les restes d'une cruche non calcinée, brisée en plusieurs fragments dispersés (Blaizot, Bonnet, 2007). L'objet est incomplet, ce qui suggère, là encore, qu'il fût brisé hors de la fosse et que seule une partie des fragments fut rejetée sur les résidus.

Enfin, le bris peut être limité à l'enlèvement du bord correspondant à un geste de décolletage, comme c'est le cas de la grande cruche posée sur la panse dans le bûcher 176 du Valladas (Bel et al., 2002, p. 247 et fig. 250).

\section{AUTRES DÉPÔTS D'OBJETS BRISÉS ET NON BRÛLÉS}

Les cruches ne sont pas les seuls objets à avoir été brisés avant leur dépôt sur les résidus de crémation des bûchers. Au Valladas, le gobelet en céramique du bûcher 105 (Bel et al., 2002, p. 457) et le balsamaire en verre du bûcher 243 ont fait l'objet d'un traitement similaire. Le fond de ce dernier récipient est placé à l'envers à distance du col, les deux fragments ayant été dissociés avant leur dépôt. Dans la même fosse se trouve également un miroir à boîtier également séparé de son couvercle, placé à distance (Bel et al., 2002, p. 147-148 et p. 445). Dans le bûcher voisin (280), le miroir retrouvé en position verticale contre la paroi est séparé du boîtier, posé à plat sur les résidus. Dans l'ensemble funéraire daté du I ${ }^{\mathrm{er}}$ s. apr. J.-C. au Sextant, rue du Commandant-Charcot à Lyon, un petit vase en bronze, percé, est situé au sommet de la couche de résidus (fig. 116).

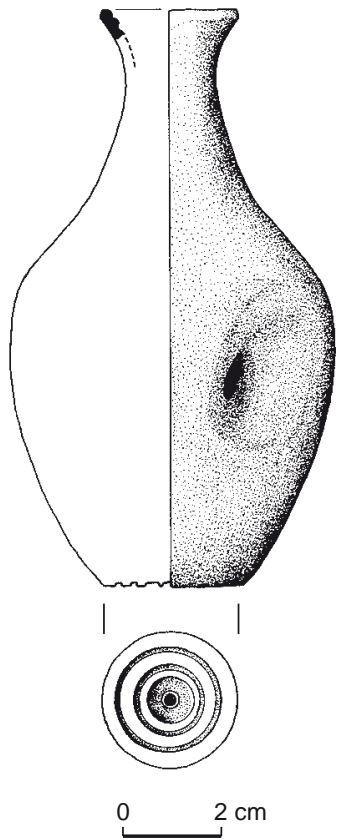

Fig. 116 - Vase en bronze non brûlé et intentionnellement percé, placé au sommet de la couche de crémation d'un bûcher du Sextant, rue du Commandant-Charcot à Lyon ( $I^{\text {er }}$ s.) (dessin : C. Plantevin, Inrap).

L'amphore du bûcher 255 de la Favorite est dépourvue de son col et d'une de ses anses (Tranoy, 1995b, p. 121 et pl. 71).

\section{LES DÉPÔTS DE MOBILIER NON BRÛLÉ ET NON BRISÉ SUR LES BÛCHERS EN FOSSE SANS OSSUAIRE}

Nombre de bûchers renferment des dépôts non brûlés et non brisés, déposés à la surface de la couche de crémation. Précisons que les bûchers dont il est question n'ont pas été utilisés pour déposer le vase ossuaire a posteriori.

Ces dépôts sont de types variés. Les données disponibles témoignent en fait de situations très diverses selon les sites et les régions. Contrairement à Lyon et à l'Auvergne, on observe qu'au Valladas et en Languedoc, la fréquence des dépôts secondaires de vases est presque aussi importante dans les bûchers sans ossuaire (respectivement $84 \%$ et $55 \%$ ) que dans les structures de dépôt avec ossuaire (95\% et $69 \%$ ). En revanche, les objets personnels n'apparaissent presque jamais dans ce type de dépôt, sauf dans ceux du Valladas où on les rencontre dans 10 bûchers datés du dernier tiers du $\mathrm{I}^{\mathrm{er}} \mathrm{s}$. et du $\mathrm{II}^{\mathrm{e}} \mathrm{s}$. Il s'agit d'objets de toilette (miroirs, strigiles, rasoir), de parure (épingles) ou des outils et instruments (stylets, serpette). À la Favorite, un 


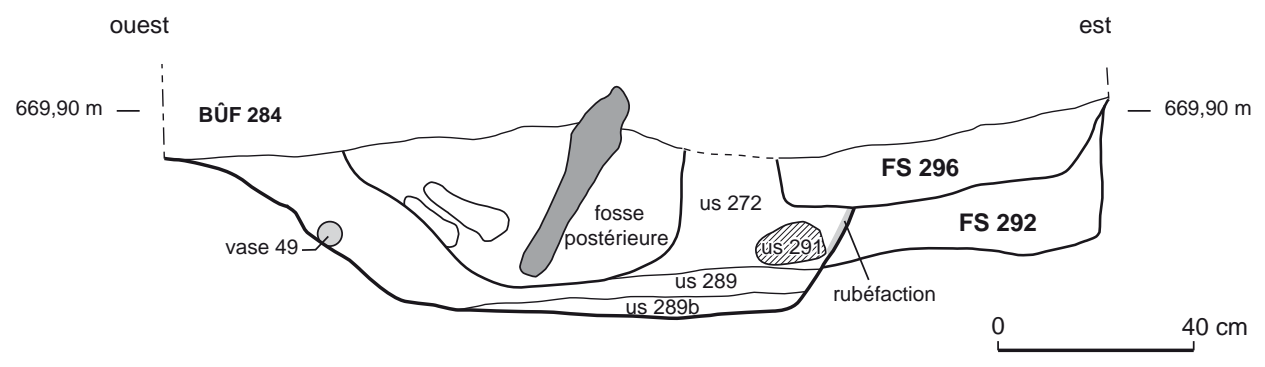

Fig. 117 - Recreusement de la couche de crémation et dépôt d'un vase du bûcher 284 du Replat à Aime, Savoie (relevé : F. Blaizot, Inrap; DAO : V. Bastard, Inrap).

coffret d'oculiste repose sur la couche de crémation du bûcher 107 (Tranoy, 1995b, p. 782). Le dépôt de faune non brûlé est également exceptionnel dans nos régions et les seules attestations, à notre connaissance, proviennent du Valladas : dépôts de quartiers de porc (bûchers 176 et 218), de gallinacé, escargots et coquillage (bûcher 45), dans des contextes du $\mathrm{I}^{\mathrm{er}} \mathrm{s}$., restes de bovidés et d'équidés dans des fosses du $\mathrm{II}^{\mathrm{e}} \mathrm{s}$. Aucun exemple n'est relevé plus au nord ou en Auvergne.

Les dépôts de vases sont les plus fréquents. La cruche est souvent concernée. Sur le site de la rue des Granges, le bûcher 48 conserve, au sommet de la couche de résidus, une cruche non brûlée dont le taux de représentation est estimé, en raison de son émiettement, entre $30 \%$ et $60 \%$. Au Replat, à Aime, une cruche représentée à $80 \%$ repose sur le bord de l'excavation qui correspond au prélèvement des os du bûcher 165. À la Favorite, $56 \%$ des bûchers datés de la première moitié du $\mathrm{I}^{\mathrm{er}} \mathrm{s}$. comprennent au moins un dépôt de cruche non brûlé. Ce chiffre chute de manière vertigineuse dans la seconde moitié du $\mathrm{I}^{\mathrm{er}} \mathrm{s}$. où seulement 2 bûchers sur 43 (493 et 462) comportent une cruche.

Au Valladas, 16 bûchers sur les 38 dépourvus d'ossuaire et, en Languedoc, 6 bûchers sur les 20 recensés livrent un dépôt secondaire de vases composé d'au moins un récipient pour les liquides (cruches, gobelets...). Le bûcher de Gallière à Montpellier renferme un gobelet complet à paroi fine dépourvu de trace de chauffe, posé à l'envers au sommet du comblement, ce qui place son dépôt au moment de la condamnation de la fosse par apport de terre. Si le dépôt secondaire de vase à liquides dans les bûchers de Vernègues apparaît exceptionnel, à Marseille, les rares dépôts secondaires de céramiques présents (9\% des cas) sont principalement des gobelets en céramique commune.

Les dépôts secondaires de balsamaires dans les bûchers s'avèrent très fréquents, par exemple dans la première moitié du I ${ }^{\mathrm{er}}$ s., à la Favorite (56\%), où ils sont généralement associés aux cruches. Dans trois bûchers de la seconde moitié du $\mathrm{I}^{\mathrm{er}}$ s. au 62 avenue du Point-du-Jour (dont deux restent non étudiés), ils sont néanmoins isolés. Au Valladas, la proportion de balsamaires en dépôt secondaire dans les bûchers est identique à celle des vases pour les liquides (16 sur 38), le vase à parfum étant souvent associé aux cruches. Dans les bûchers 176 et 218 par exemple, ces objets sont regroupés avec des vases pour les liquides, contrairement à ce qui est observé dans les dépôts primaires où ils apparaissent dispersés ou associés aux vases pour les solides (Bel et al., 2002, p. 143). Dans d'autres cas, le balsamaire accompagne la lampe ou des objets personnels. En LanguedocRoussillon, le dépôt secondaire de balsamaire n'apparaît que dans 4 bûchers sur 20 en association avec les vases à liquide ou les lampes. À Vernègues, les dépôts secondaires de mobilier semblent essentiellement constitués de balsamaires (présents dans 17 bûchers sur 45) et de lampes (dans 16 cas). La lampe apparaît fréquemment en dépôt secondaire dans les bûchers du Valladas, puisque les deux tiers (24 sur 38) en livrent au moins une.

En Rhône-Alpes, les dépôts secondaires de lampe dans les bûchers sont rares : on en compte deux à la Raude et une au 62 avenue du Point-du-Jour. Dans le premier cas, elles sont placées en bordure de l'une des cuvettes qui résultent du réaménagement du bûcher, et plus précisément de celle qui contient les os. Dans le second, la lampe a été placée à l'envers, au sommet de la couche de résidus. Parmi tous les bûchers de la Favorite, un seul livre une lampe, mais le caractère secondaire du dépôt est douteux (bûcher 31).

Deux bûchers du Replat à Aime, un du 54 rue PierreAudry, un voie de l'Océan et quatre à la Favorite renferment un pot en céramique commune dont la fonction dans ce cadre précis reste indéterminée. Au Replat, on les trouve encore à l'endroit où la couche de résidus a été recreusée (fig. 107, vase 57 et fig. 117, vase 49) ; rue Pierre-Audry, le 
pot repose au sommet de la couche de résidus. Le dépôt secondaire de vases à solides est peu attesté en Languedoc : deux bûchers de la Céreirède à Lattes livrent des assiettes, un couvercle et un pot non brûlés. En revanche, la fréquence de ces dépôts est identique à celle des vases pour les liquides dans les bûchers du Valladas (16 cas sur 38).

\section{LE DÉPLACEMENT DU MOBILIER PRIMAIRE}

Quelques observations ponctuelles signalent des déplacements de mobilier brûlé au sein même du bûcher. Ces objets sont retrouvés groupés au sommet de la couche de résidus, alors que les autres éléments du dépôt primaire sont inclus dans la couche charbonneuse ou posés sur le fond de la fosse. Citons par exemple les deux balsamaires, brûlés mais intacts, du bûcher 45 de la rue des Granges à Lyon ou le plat brûlé du bûcher 5 de l'Estel, retrouvé à l'envers à la surface de la couche de résidus. Dans le bûcher 209 du Valladas, le fond d'une cruche brûlée a servi de couvercle à un pot non brûlé déposé sur les résidus de la crémation (Bel et al., 2002, p. 340).

Des fragments d'objets brûlés sont rencontrés dans le comblement des fosses au Valladas et à Nîmes. C'est aussi le cas de l'armature de pied de lit mise au jour en position oblique dans le remblaiement du bûcher 6200 de SaintFréjus à Marennes, les trois autres étant restés dans les profondeurs de la couche de résidus. Dans le bûcher de Peyre Plantade, trois des armatures de pieds de lit ont été regroupées en faisceau le long d'une paroi de la fosse. À la Favorite, L. Tranoy parle de "dépôt primaire déplacé », en l'occurrence au sommet des résidus, pour un balsamaire du bûcher 295 (Tranoy, 1995b, p. 289). Au sommet du comblement définitif du bûcher en fosse des Sables à Limé (Aisne) se trouve un lot de vases constitué d'un bol non brûlé et, à l'autre extrémité de la fosse, d'un empilement, de douze récipients et de quatre lampes calcinés provenant de toute évidence du mobilier primaire (Soupart, 2001, p. 71).

Ces exemples indiquent qu'une partie du mobilier primaire semble avoir été réinvestie dans une étape différente du rituel, à moins que ces situations ne transcrivent qu'un geste anecdotique ; dans le cas précis du bûcher des Sables, les céramiques auraient alors été redéposées de cette manière, sans raison précise, à l'occasion des remaniements liés aux prélèvement des os suggérés par la faible masse osseuse en présence (274 g). L'étude de la répartition spatiale des régions anatomiques indique en effet que les ossements sont complètement mélangés (Soupart, 2001, p. 72), tandis que la figure 10 montre que la céramique est rassemblée dans le quart sud-est de la fosse. On se trouve peut-être ici dans le cas évoqué plus haut (voir supra, p. 158-159), dans lequel les résidus sont exhumés le temps de récolter les os, puis reversés dans la fosse ; les céramiques calcinées auraient été rassemblées et empilées dans un coin avant de combler définitivement la fosse.

Dans tous les cas, la difficulté est de distinguer ce qui relève des remaniements liés à la récupération des restes osseux (voir chapitre IV, p. 183-186) du geste intentionnel raisonné. En outre, il est possible que certains objets se retrouvent à la surface de la couche de crémation du fait des pratiques de conduction du bûcher ; de ce fait, seuls les empilements ou regroupements de vases sur une surface limitée s'avèrent significatifs.

\section{INTERPRÉTATION DES DÉPÔTS}

Quelle que soit leur situation, dans les bûchers ou dans les structures de dépôt, les objets non brûlés ont longtemps été interprétés comme des «viatiques " faits au mort, exprimant « la croyance en une forme de survie dans l'au-delà »(Bel et al., 2002, p. 111). Or, les textes antiques n'évoquant en aucun cas une telle fonction du mobilier, quel qu'il soit, et la conception de « l'au-delà » des Romains s'opposant totalement à toute jouissance, cette appellation peut être rejetée sans nuance. Comme le rappellent S. Lepetz et W. Van Andringa, le terme de munus qui apparaît dans les textes antiques, signifie que le mobilier a valeur d'hommage (Lepetz, Van Andringa, 2004). Sans doute faut-il considérer que chaque élément du mobilier connaît des destinations symboliques différentes au cours des différentes étapes de l'ensemble du rite funéraire, et la prise en compte de plusieurs paramètres (choix fonctionnel des dépôts, situation dans la structure et modalités de dépôts conjugués à une révision du statut des structures) ouvre des perspectives d'interprétation plus diversifiées (Blaizot, Bonnet, 2007).

Les deux modes de dépôt de la cruche (ou de l'amphore), l'une brisée dont on rejette certains fragments et l'autre complète, n'appartiennent pas forcément au même registre. Quoi qu'il en soit, ces cruches et ces amphores ne sont par ailleurs probablement que les instruments, laissés sur place, d'un rite manifestement dédié au versement des liquides. La fonction des pots est-elle différente ? Rien ne prouve qu'ils étaient, dans ce cas précis des funérailles, destinés aux solides puisque, dans le cadre funéraire, la fonction habituelle des objets peut très bien être modifiée. D'autres dépôts comme les plats et les assiettes, attestés au 
Valladas et à Lattes, ou les restes de faune (dont des pièces en connexion), se rapportent néanmoins indiscutablement aux nourritures solides.

Ces dépôts ne sont pas relevés dans toutes les structures, mais s'avèrent au contraire minoritaires. Ce caractère non systématique pose a priori un problème. Les rites qui leur sont liés étaient-ils réservés à une catégorie d'individus ? Aucun argument ne permet de le démontrer et l'hypothèse selon laquelle le rite se faisait systématiquement, mais qu'il n'y avait aucune obligation particulière à laisser les instruments sur place, en tout cas dans la structure, apparaît plus plausible.

\section{LES DISPOSITIFS DE FERMETURE ET DE CONDAMNATION DES BÛCHERS}

\section{LE SCELLEMENT DES BÛCHERS}

Le plus souvent les bûchers en fosse sont remblayés avec de la terre souvent mêlée de résidus charbonneux (Bel et al., 2002, p. 95 ; Chapon et al., 2004, p. 140). Dans plusieurs exemplaires du Valladas comme dans le bûcher 12 de la ZAC Sextius-Mirabeau, on distingue nettement deux apports de terre successifs (Bel et al., 2002, p. 95 ; Nin et al., 2006, p. 176), ce qui implique un remblaiement en deux étapes. Un dépôt de mobilier non brûlé est parfois mis en place sur la première couche qui surmonte la couche de crémation : un gobelet, une serpette et un balsamaire dans le bûcher 105 et un balsamaire dans le bûcher 136bis. En général, la couche inférieure comporte plus d'inclusions charbonneuses que le remblai supérieur. Dans les bûchers B et 137, les résidus ont été scellés par une couche d'argile verte, avant de remblayer la fosse (Bel et al., 2002, p. 78 et p. 95). Dans le bûcher B, dont les parois étaient maçonnées, la couche d'argile était associée à des moellons provenant de la destruction des parements de la fosse (Bel et al., 2002, p. 357). Ces dépôts d'argile verte spécifiques à l'ensemble funéraire du Valladas apparaissent également dans plusieurs structures secondaires où ils recouvrent l'ossuaire ou le dépôt de mobilier et dans des fossés contenant des dépôts d'équidés. L'argile utilisée ne provient pas du site mais de formations proches de Saint-Paul-Trois-Châteaux (Bel et al., 2002, p. 101-102). L'auteur envisage qu'elle fût apportée expressément pour sceller chaque dépôt et propose que des vertus particulières lui étaient attribuées, justifiant sa place dans les pratiques funéraires au moment de la fermeture des structures, bûchers ou structures de dépôt.

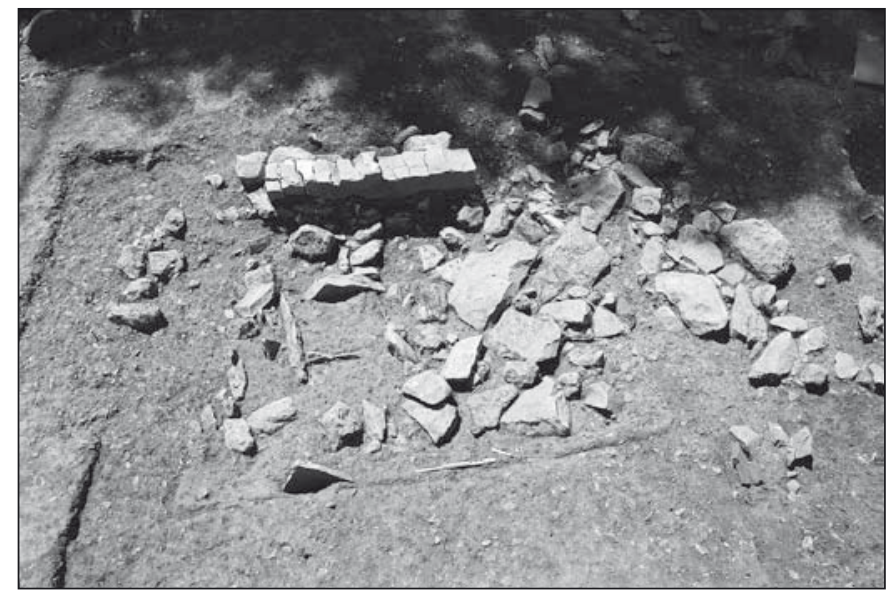

Fig. 118 - Amas de blocs sur les résidus du bûcher 1055 du 78 avenue Jean-Jaurès à Nîmes, Gard (cliché : B. Thuillier).

De nombreux exemples relevés en Rhône-Alpes montrent que le remblaiement se faisait assez rapidement après la crémation et qu'il n'atteignait pas forcément le haut de la fosse. En effet, des effondrements de la partie supérieure rubéfiée des parois sont relevés en surface de la couche de comblement du bûcher qui surmonte les résidus. L'épaisseur de ces couches $(40 \%$ à $60 \%$ du remplissage au quai Arloing et $70 \%$ du bûcher 166 de la rue du Chapeau-Rouge, par exemple) relativement au court temps nécessaire à la partie rubéfiée de la paroi pour s'effondrer, nous invite à rejeter l'hypothèse d'un comblement naturel. Toutefois, lors de sondages récemment pratiqués au 62 rue du CommandantCharcot, les auteurs ont noté que la couche d'effondrement des parois reposait directement sur celle des résidus, et donc sous le sédiment hétérogène correspondant au comblement définitif de la fosse. On rencontre finalement assez souvent des plaques de parois rubéfiées effondrées sur les résidus, voire, comme au Valladas, sur des dépôts de vases non brûlés (Bel et al., 2002, p. 95). Si cette configuration n'est pas due à la présence initiale d'une couverture en matériau organique, cela signifierait que, dans certains cas, la fosse n'était pas remblayée, mais cette proposition doit être pondérée par le fait que des parois rubéfiées s'effondrent facilement lors du refroidissement de la fosse.

Le scellement des fosses de crémation peut être constitué d'une couche de blocs comme dans le bûcher 1055 du 78 avenue Jean-Jaurès à Nîmes (fig. 118). L'amas de blocs avait dans ce cas été installé après la mise en place sur les résidus d'un petit coffrage de dalles abritant le dépôt secondaire. La bonne conservation de la structure a permis de constater que le remplissage de pierres débordait au-dessus du sol et constituait ainsi un petit tertre sur la fosse. 


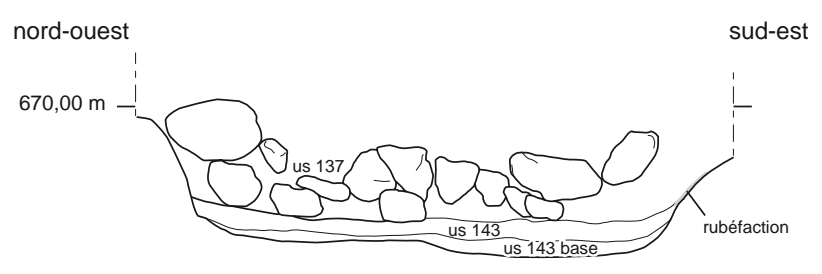

BÛF 147

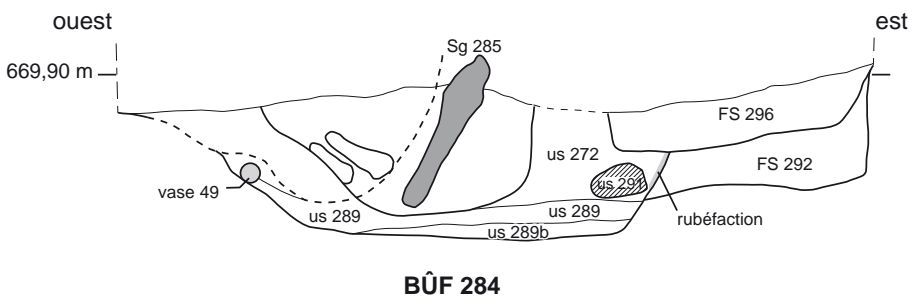

$\underline{0} \quad 50 \mathrm{~cm}$

Fig. 119 - Chape de blocs sur le bûcher 147 du Replat à Aime, Savoie (relevé : F. Blaizot, Inrap ; DAO : V. Bastard, Inrap).

Des blocs isolés sont fréquemment signalés à la surface des couches de crémation. Si certains peuvent être attribués à un dispositif de couverture (voir infra, p. 172), la plupart ne semblent pas avoir de rôle fonctionnel, comme le fragment de marbre trouvé dans un bûcher du site de l'Hôtel-Dieu à Narbonne (Ginouvez dir., 1996-1997, p. 128) ou les blocs de grès et de calcaire rougis à la base dans le bûcher 2247 du Mas de Vignoles IX à Nîmes. Il a été proposé de les interpréter comme des éléments symboliques marquant le scellement de la structure (Ginouvez dir., 1996-1997, p. 128).

Dans trois bûchers du Replat à Aime, une chape de blocs repose en partie sur la couche de résidus et en partie en suspension dans le comblement supérieur (fig. 119). Il reste difficile de savoir si cette situation est celle d'origine ou si elle transcrit l'effondrement d'une superstructure. Si l'on retient la première hypothèse, il faut supposer que ces blocs ont été mis en place un certain moment, certes peu mesurable, après la crémation, puisque des plaques de sédiment rubéfié provenant de l'effondrement des parois sont intercalées entre les résidus et les blocs. Dans la seconde hypothèse, nous devons envisager la présence d'une couverture en matériau périssable sur toute la surface du bûcher, qui seule peut expliquer la chute des blocs directement sur la couche de crémation.

\section{LES DISPOSITIFS DE COUVERTURE}

Plusieurs découvertes méridionales illustrent des dispositifs de fermeture assez comparables à ceux que l'on rencontre dans les inhumations. Les exemples les plus spectaculaires proviennent du site de Sainte-Barbe, où les couvertures en dur sont conservées (Chapon et al., 2004, p. 115-117 et p. 140). Ainsi, le bûcher 68 est fermé par une dalle taillée dans une pierre de pressoir qui repose sur des ressauts aménagés dans les parois de la fosse (Chapon et al., 2004, p. 190). Le bûcher 6 est recouvert par une série de quatre tegulae à plat placées sur le sommet de la fosse, sans doute cette fois après remblaiement, puisque la couverture est plus étroite que la fosse (id., ibid., p. 147), à moins d'envisager un dispositif de bois sur lequel reposaient les tuiles. La couverture est parfois installée dans la fosse, sur les résidus ; c'est le cas des bâtières de tuiles déjà mentionnées de Fréjus (Béraud et al., 1985, p. 13), Narbonne (Dellong, 2002, p. 333) et Sainte-Barbe (Chapon et al., 2004, p. 171 et p. 193, fosses 40 et 69). Les couvertures horizontales reposent, quant à elles, le plus souvent sur des éléments de calage placés sur la couche de crémation, à l'instar de la dalle de marbre de la fosse 65 de Sainte-Barbe ou des tegulae de la fosse F67 (Chapon et al., 2004, p. 187-189). Les calages sont constitués de blocs localisés le long des parois de la fosse (id., ibid., p. 188) ou de fragments de tuiles (fosse 67 : id., ibid., p. 189) ; dans certains cas, ce sont les seuls éléments qui subsistent du dispositif (fosses 48 et 71 : id., ibid., p. 180 et p. 194). À Nîmes, le bûcher 2249 du Mas de Vignoles IX conserve, sur la couche des résidus, trois blocs dont un élément en position oblique et une dalle placée sur chant, dont la partie sommitale, brisée, se trouve posée à plat à proximité. Cette disposition implique que la pierre a été fracturée avant son dépôt ou bien qu'elle s'est effondrée en place en effectuant une rotation sur elle-même, au sein d'un 
espace vide. Cette dernière hypothèse est confortée par la position du bloc de calcaire oriental qui a manifestement basculé au cours du colmatage de la tombe. La nature du comblement apporte un argument supplémentaire. Il est en effet constitué à la base d'un sédiment argileux formé de lits successifs résultant très probablement d'un colmatage par infiltration. Ces indices suggèrent de restituer un dispositif de fermeture en matériau périssable reposant sur les calages de pierre. Des traces ligneuses de bois non brûlé, associées à des clous, ont été observées " au-dessus de la couche de crémation ", dans le bûcher 136 du site de Sainte-Barbe à Marseille (Moliner et al., 2003, p. 300).

Ces dispositifs de couverture sont relevés dans des bûchers dépourvus d'ossuaire, dont certains comportent une masse d'os importante (2 $043 \mathrm{~g}$ dans l'exemplaire de Sainte-Barbe, 1 102,7 g dans celui de Nîmes, 1 042,5 g dans la fosse 48 de Sainte-Barbe), alors que d'autres ont fait l'objet de prélèvements comme à Sainte-Barbe (bûcher 68 : 939,7 g ; bûcher $65: 547,7 \mathrm{~g}$; bûcher $40: 442,5 \mathrm{~g}$; bûcher $71: 317,7 \mathrm{~g}$; bûcher $67: 9,9 \mathrm{~g})$.

Aucun bûcher de Rhône-Alpes n'atteste clairement ce type d'aménagement. Dans le bûcher 3041 des 77-79 rue Pierre-Audry, on a vu que les données du rapport ne permettent pas de rapporter ces tuiles à la construction ou à la fermeture du bûcher (voir chapitre II, p. 109-110).

Les configurations s'avèrent une fois de plus très diverses : des bûchers paraissent avoir été comblés assez vite, sans doute à l'extrême fin de la cérémonie et d'autres furent protégés par une couverture, parfois après avoir été en grande partie vidés. Était-il prévu de les réutiliser? On peut aussi mettre ces différences en relation avec le statut particulier que peuvent acquérir certaines de ces structures (voir chapitres IV et VI).

\section{LES CONDUITS À LIBATION SUR LES BÛCHERS}

Des sites méridionaux offrent des exemples indiscutables de dispositifs destinés à mettre en relation la surface du sol et la couche de crémation ou le vase ossuaire éventuellement placé sur cette dernière. Il s'agit le plus souvent d'un conduit cylindrique en terre cuite incomplet de $30 \mathrm{~cm}$ de longueur, disposé verticalement, parfois de col d'amphores placés à l'envers au contact ou légèrement au-dessus des résidus charbonneux. Les dispositifs de couverture des bûchers en fosse 6, 67 et 69 de Vernègues présentent ainsi un orifice surmonté par des restes d'amphores : cols ou fonds (Chapon et al., 2004, p. 147, 189, 193). Il est possible aussi que l'axe central de la meule en remploi utilisée pour la fermeture

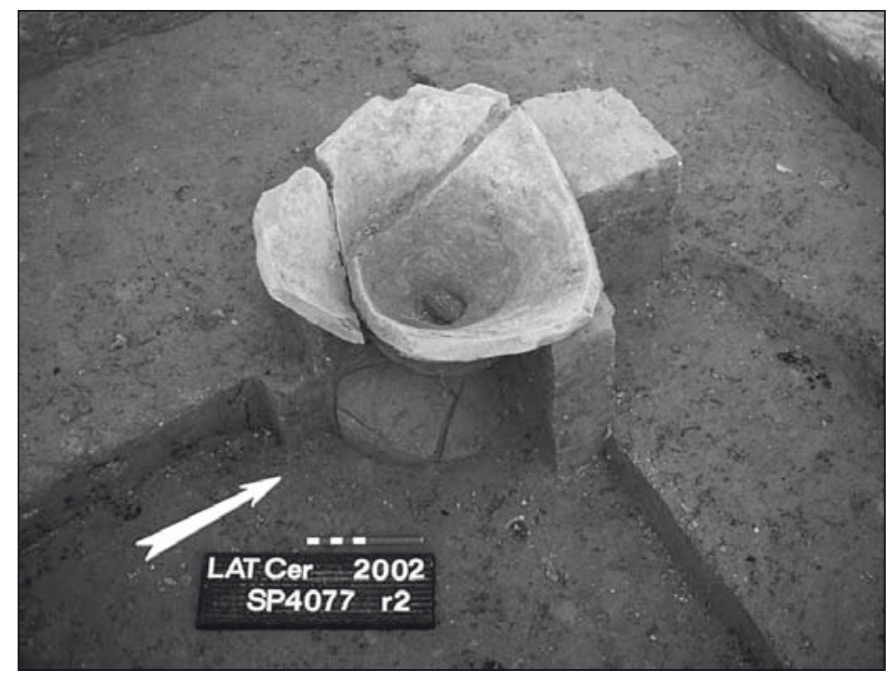

Fig. 120 - Vue du fond d'amphore percé placé sur un col de cruche en dépôt secondaire dans le bûcher 4077 de la Céreirède à Lattes, Hérault (cliché : G. Loison, Inrap).

du bûcher 68 ait eu un usage similaire (id., ibid., p. 191). En Languedoc-Roussillon, l'exemple le plus explicite provient de l'ensemble funéraire de la Céreirède ; un fond d'amphore Gauloise percé repose sur le col d'une cruche non brûlée placée en position fonctionnelle sur les résidus du bûcher 4077 (fig. 120). Le bûcher 10 de Vers-Pont-du-Gard livre, quant à lui, deux imbrices qui traversent en position oblique le comblement. Elles reposent l'une sur l'autre, face convexe vers le haut, la base sur les restes de la crémation, contre un fond d'amphore Gauloise en position fonctionnelle. Elles peuvent être interprétées comme des éléments de conduits à libation ayant basculé au cours du colmatage de la fosse, ce qui suppose l'existence d'une couverture. Cette hypothèse est également suggérée par l'affaissement des fragments de paroi de l'amphore à l'extérieur du contenant et par un sédiment très limoneux à la base du remplissage. À ces exemples, se rajoutent les nombreuses amphores observées dans le comblement des bûchers en fosse, au-dessus de la couche de crémation à Sainte-Barbe (Chapon et al., 2004, p. 116-117), et à Lattes, sur le site de la Céreirède. Ces éléments se trouvant cependant à distance de la couche de crémation, il est difficile de déterminer si l'on a affaire à des conduits à libation ou à des dispositifs de signalisation.

Les conduits avérés concernent des bûchers sans ossuaire qui ont fait l'objet de prélèvements d'os ou qui ont été complètement vidés, comme les bûchers 4 (90,5 g d'os), 59 $(5,6 \mathrm{~g}), 63$ (12,6 g) et 67 (9,9 g) à Sainte-Barbe, ou qui ont au contraire livré des masses d'os importantes (le bûcher 10 de Vers-Pont-du-Gard contenait 1 953,2 g d'os brûlés). 


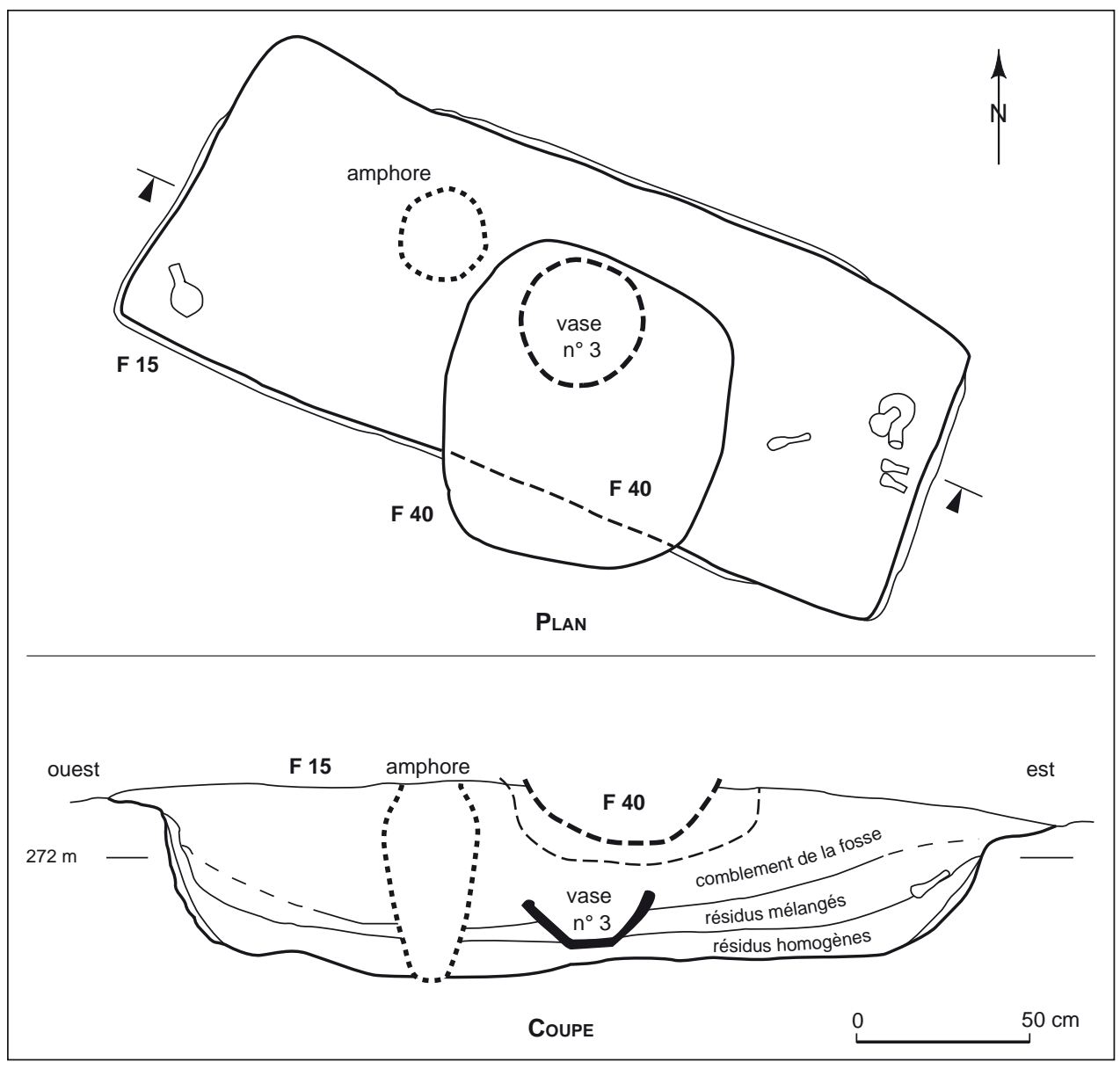

Fig. 121 - Installation d'un ossuaire (vase $n^{0} 3$ ) dans la couche de résidus du bûcher 15 du 41 rue Joliot-Curie à Lyon (relevé : A. Bouvier ; DAO : G. Macabéo, Inrap).

À Lyon, en Rhône-Alpes et en Auvergne, les conduits à libations sont plutôt recensés dans les structures de dépôt (voir chapitre IV), et deux exemples relevés en contexte de bûcher à Lyon restent à discuter. Le premier, au 41 rue Joliot-Curie, concerne une amphore dont l'épaule et le fond sont tronqués, plantée verticalement dans les résidus qui tapissent le fond de la fosse, et contre laquelle viennent buter les couches du comblement supérieur (fig. 121). Toutefois, ce bûcher nous pose un problème dans le sens où il comporte, enfoncé à la surface de la couche de crémation, une coupe dont le contenu non fouillé demeure un mystère : s'agit-il d'un vase ossuaire ou d'un dépôt secondaire de vase ? Dans le premier cas, on pourrait subodorer que l'amphore est en relation avec l'ossuaire, bien que les deux éléments se jouxtent. Le deuxième exemple est illustré par le bûcher 1000 du 62 avenue du Point-du-Jour, dans lequel se trouvaient les restes d'une amphore Dressel 20, qui traverse les comblements supérieurs de la fosse et aboutit au sommet de la couche de crémation. L'hypothèse qu'elle ait pu servir de conduit à libation ne peut être démontrée dans la mesure où elle n'est représentée que par quelques fragments, dont des éléments du col dispersés à la surface des résidus (fig. 122). Ces vestiges se rapportent tout aussi bien à des bris rituels de vases à liquide au sommet de la couche de crémation (voir supra, p. 164-167).

Les conduits à libations étaient probablement plus nombreux à l'origine ; les dispositifs relevés dans les structures secondaires laissent en effet prévoir l'emploi de matériaux périssables pour remplir cette fonction de libation (voir chapitre IV). L'implantation d'un conduit à libation dans des bûchers qui ne comportent pas d'ossuaire et dans lesquels la couche de résidus ne fait pas l'objet d'un réagencement et où la masse osseuse est très déficitaire, pose le problème de la signification de ces pratiques et donc du moment où elles interviennent. Est-ce un dispositif destiné à servir une seule fois pour consacrer l'emplacement de la 
crémation ou fut-il utilisé sur la durée ? Dans le second cas, que commémorent ces libations ? Ces questions, à l'heure actuelle sans réponse, sont à mettre là encore en relation avec la question du statut de ces structures (voir chapitre VI) et les problèmes de conservation du dispositif général (ossuaires aériens non conservés).

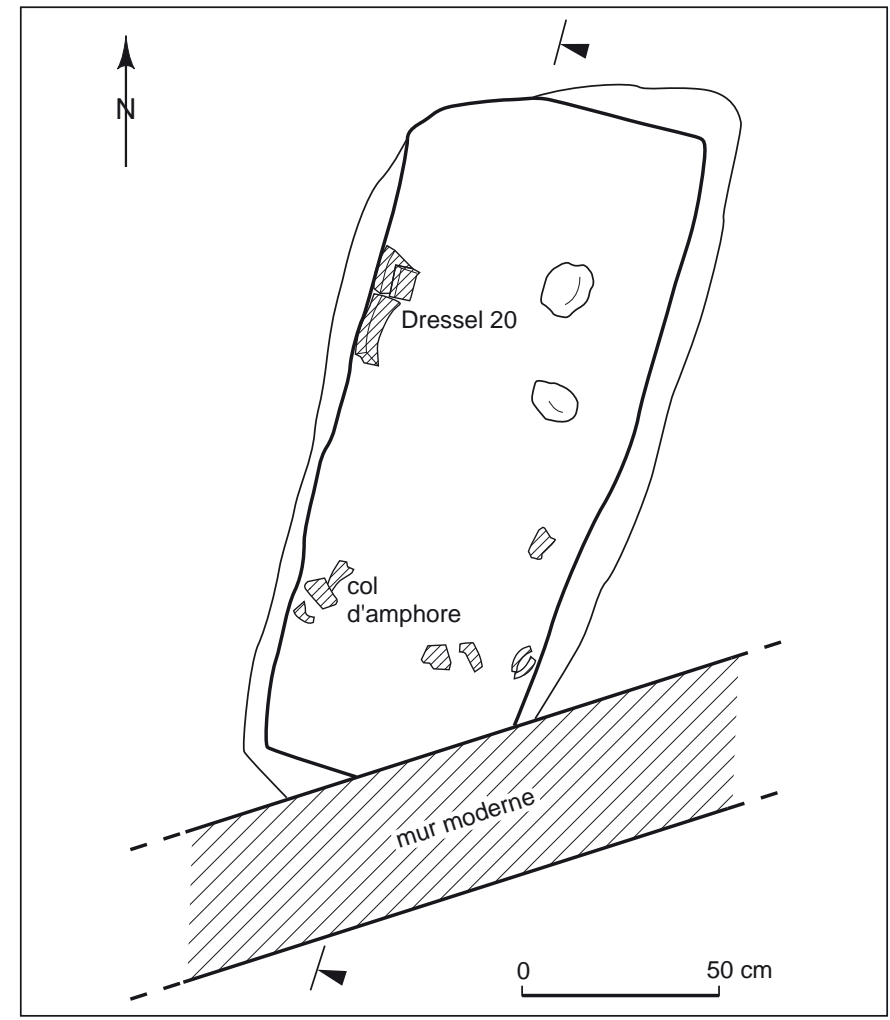

Fig. 122 - Amphore Dressel 20 au sommet du bûcher 1000,

62 avenue du Point-du-Jour à Lyon (relevé : L. Kuntz, Inrap; DAO : G. Macabéo, Inrap). 